\title{
Astrometric radial velocities
}

\section{Hipparcos measurements of nearby star clusters and associations ${ }^{\star, \star \star}$}

\author{
Søren Madsen, Dainis Dravins, and Lennart Lindegren
}

Lund Observatory, Box 43, 22100 Lund, Sweden

e-mail: soren, dainis, lennart@astro.lu.se

Received 10 September 2001 / Accepted 15 October 2001

\begin{abstract}
Radial motions of stars in nearby moving clusters are determined from accurate proper motions and trigonometric parallaxes, without any use of spectroscopy. Assuming that cluster members share the same velocity vector (apart from a random dispersion), we apply a maximum-likelihood method on astrometric data from Hipparcos to compute radial and space velocities (and their dispersions) in the Ursa Major, Hyades, Coma Berenices, Pleiades, and Praesepe clusters, and for the Scorpius-Centaurus, $\alpha$ Persei, and "HIP 98321" associations. The radial motion of the Hyades cluster is determined to within $0.4 \mathrm{~km} \mathrm{~s}^{-1}$ (standard error), and that of its individual stars to within $0.6 \mathrm{~km} \mathrm{~s}^{-1}$. For other clusters, Hipparcos data yield astrometric radial velocities with typical accuracies of a few $\mathrm{km} \mathrm{s}^{-1}$. A comparison of these astrometric values with spectroscopic radial velocities in the literature shows a good general agreement and, in the case of the best-determined Hyades cluster, also permits searches for subtle astrophysical differences, such as evidence for enhanced convective blueshifts of F-dwarf spectra, and decreased gravitational redshifts in giants. Similar comparisons for the Scorpius OB2 complex indicate some expansion of its associations, albeit slower than expected from their ages. As a by-product from the radial-velocity solutions, kinematically improved parallaxes for individual stars are obtained, enabling Hertzsprung-Russell diagrams with unprecedented accuracy in luminosity. For the Hyades (parallax accuracy 0.3 mas), its main sequence resembles a thin line, possibly with wiggles in it. Although this main sequence has underpopulated regions at certain colours (previously suggested to be "Böhm-Vitense gaps"), such are not visible for other clusters, and are probably spurious. Future space astrometry missions carry a great potential for absolute radial-velocity determinations, insensitive to the complexities of stellar spectra.
\end{abstract}

Key words. methods: data analysis - techniques: radial velocities - astrometry - stars: distances stars: kinematics - open clusters and associations: general

\section{Introduction}

This paper is the third of a series on the determination of stellar radial motion by purely geometric measurements. Such astrometric radial velocities allow to disentangle stellar motion from other astrophysical phenomena causing spectroscopic line shifts, such as internal motions in stellar atmospheres and gravitational redshifts. Paper I (Dravins et al. 1999b) discussed different methods which permit radial velocities to be determined independent of spectroscopy. Among these, the moving-cluster method of changing angular separation permits radial-velocity accuracies on a sub-km s${ }^{-1}$ level to be reached already with

Send offprint requests to: S. Madsen,

e-mail: soren@astro.lu.se

* Based on observations by the ESA Hipparcos satellite.

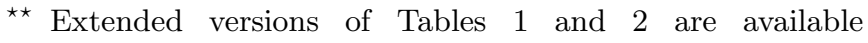
in electronic form at the CDS via anonymous ftp to cdsarc.u-strasbg.fr $(130.79 .125 .8)$ or via

http://cdsweb.u-strasbg.fr/cgi-bin/qcat?J/A+A/381/446 current astrometric measurements, such as those from the Hipparcos space mission (ESA 1997). That method analyses the changing angular extent of a star cluster as it approaches or recedes from the Sun, assuming that the member stars share the same average velocity vector relative to the Sun. The radial-velocity component makes the cluster appear to expand or contract due to its changing distance. This relative rate of apparent contraction equals the relative rate of change in distance to the cluster, from which a linear velocity (in $\mathrm{km} \mathrm{s}^{-1}$ ) follows whenever the absolute distance is known from trigonometric parallaxes.

That it is possible, in principle, to determine radial velocities from astrometry has been known for a long time. Attempts were made e.g. by Petrie $(1949,1963)$ and Eggen $(1984,1998)$ to derive radial velocities that are at least partially independent of spectroscopy. The availability of the Hipparcos results allowed to investigate the kinematics and memberships of moving groups and clusters in great detail, which in turn made it possible to apply our movingcluster method to reach better accuracies. The detailed 


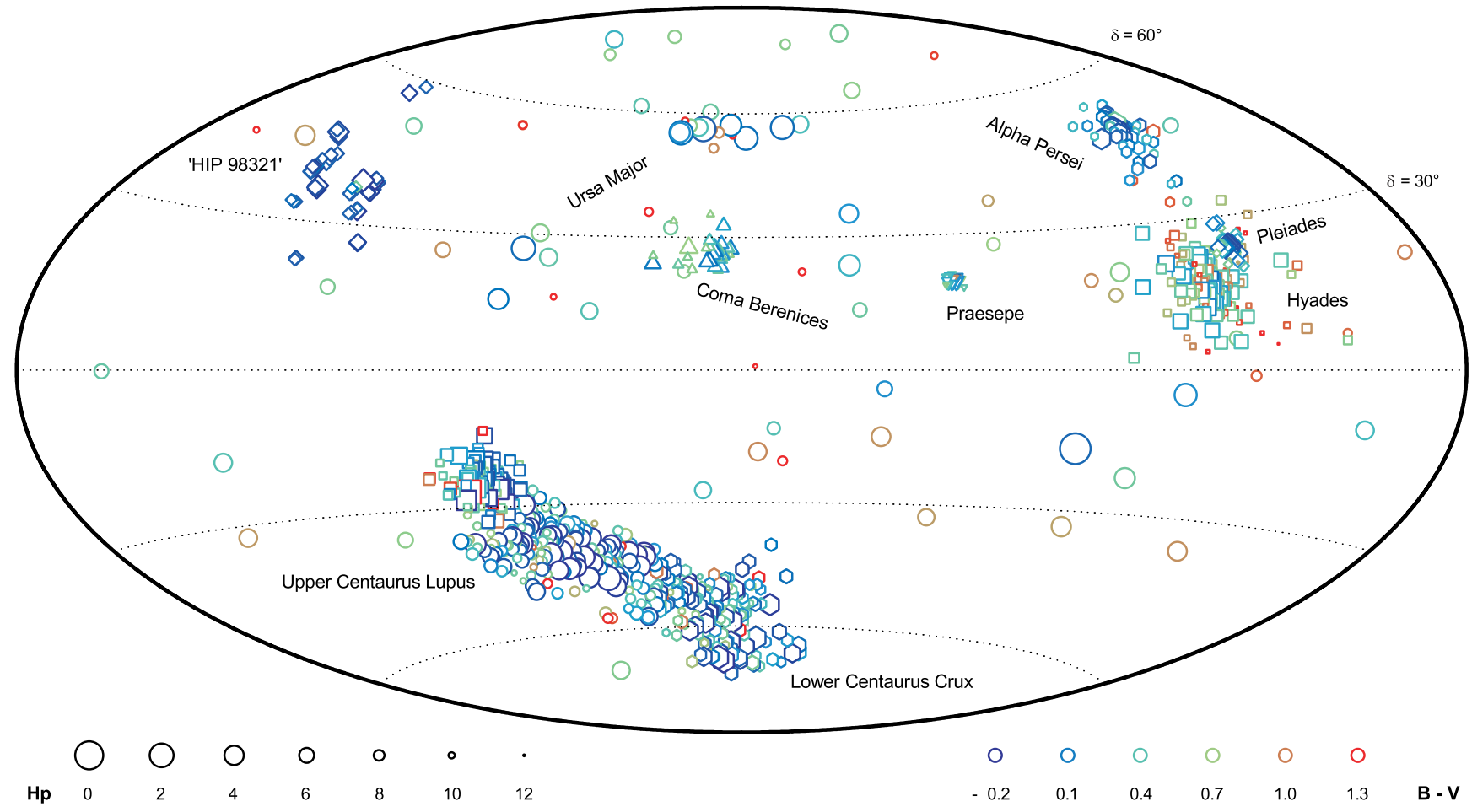

Fig. 1. Map of the full sky, showing those stars in clusters and associations, whose radial velocities were astrometrically determined from Hipparcos data. Symbol shape identifies different clusters; symbol size denotes apparent magnitude $H p\left(\simeq m_{V}\right)$, while symbol shading denotes $B-V$ (note how some clusters are dominated by very blue stars). The Aitoff projection in equatorial coordinates is used, with $\delta=0^{\circ}$ on the major axis and $\alpha=180^{\circ}$ on the minor axis. Right ascension $(\alpha)$ increases to the left.

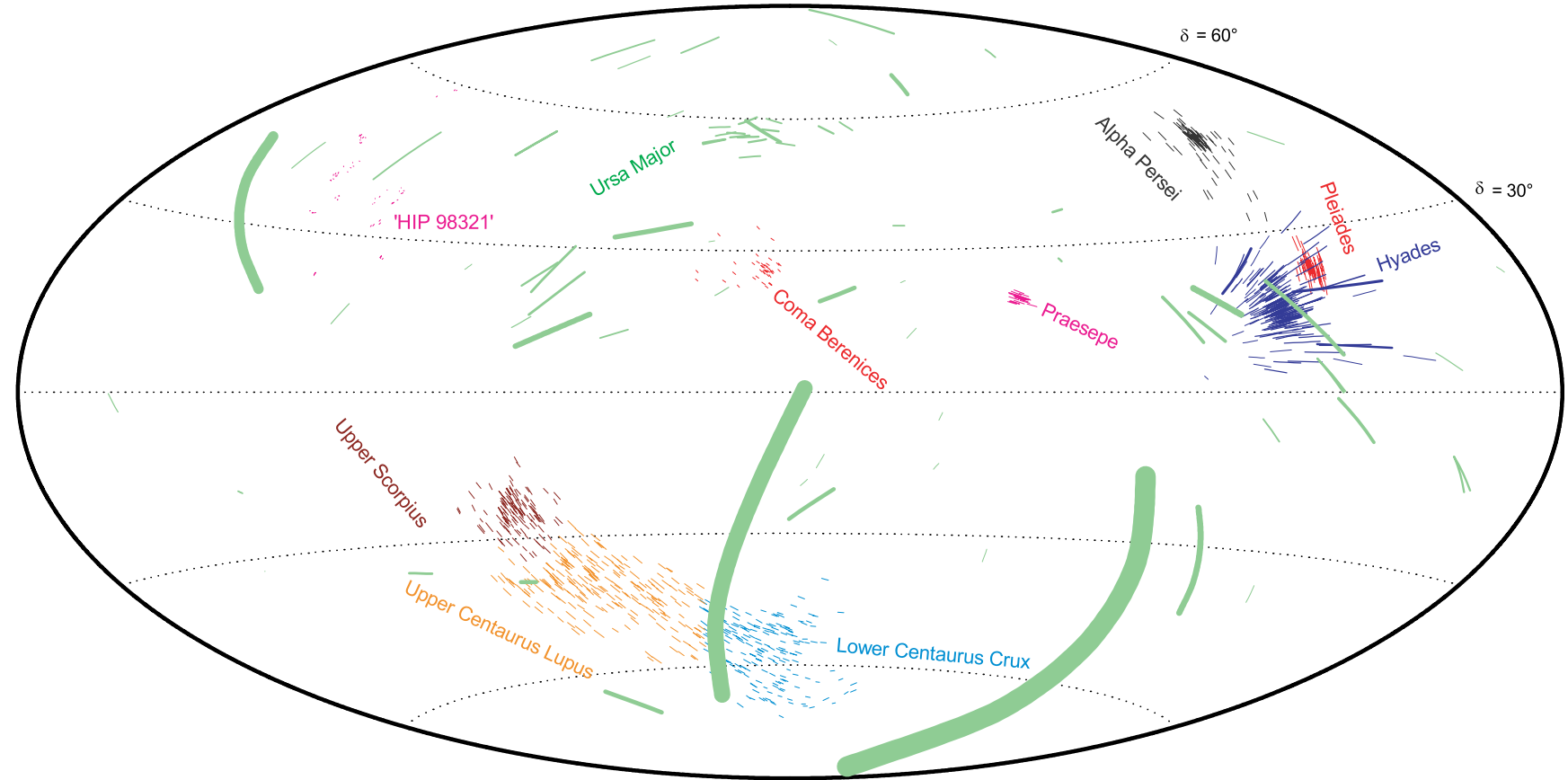

Fig. 2. Proper motions of the programme stars over 200000 years. Best radial-velocity accuracy is obtained in rich nearby clusters with large angular extent, and large proper motions. However, the accuracy in the largest associations (Ursa Major, Scorpius-Centaurus) is limited by the partly unknown expansion of these systems. Stellar paths in the Ursa Major group (shown shaded) cover large areas of the sky. The thickness of the proper-motion vectors is inversely proportional to stellar distance: the closest star is Sirius and the two next ones are faint red dwarfs. Proper motions vary greatly among different clusters. 
mathematical formulation of the method was presented in Paper II (Lindegren et al. 2000).

As a by-product of the moving-cluster method, the distance estimates to individual cluster stars are often significantly improved compared with the original [here Hipparcos] parallax measurements. These kinematically improved parallaxes (Paper II) result from a combination of trigonometric and kinematic distance information, where kinematic distances follow from the observed proper motions and the derived cluster velocity. The improved distances sometimes allow the Hertzsprung-Russell diagram of a cluster to be studied with unprecedented resolution in absolute magnitude.

In this paper we apply this "moving-cluster method" to several nearby open clusters and associations. Astrometric radial velocities and kinematically improved parallaxes are deduced for stars in the the Ursa Major, Hyades, Coma Berenices, Pleiades, and Praesepe clusters, and for the Lower Centaurus Crux, Upper Centaurus Lupus, Upper Scorpius, $\alpha$ Persei, and "HIP 98321" associations.

\section{Potential of the moving-cluster method}

The achievable accuracies of the moving-cluster method were discussed in Paper I. We recall that the best radialvelocity accuracy is obtained for star-rich nearby clusters with large angular extent, large proper motions, small internal velocity dispersions, and small rates of cluster expansion. The improved astrometric accuracies expected from future space missions will somewhat lessen these constraints, although the intrinsic limitations set by internal velocities cannot be overcome by increasing observational accuracy.

Only about ten clusters and associations can be meaningfully studied already with current astrometric accuracies in the milliarcsecond range. Furthermore, good astrometric data are available only for their relatively brighter stars. The distribution on the sky and the geometries of these nearby clusters are shown in Figs. 1 and 2, together with their stellar populations, and their proper-motion patterns. Only a few among these (Hyades, Pleiades, Coma Berenices, Praesepe) have a utilizable stellar population spanning many spectral types; several of the others are heavily dominated by early-type stars. The main reason for this is of course the limiting magnitude of the Hipparcos mission. The areas subtended on the sky differ greatly: some OB-associations spread out over much of a hemisphere, while some other clusters are very localised. Although the great spatial extents of the Ursa Major cluster and the Scorpius-Centaurus associations in principle are advantageous for the accuracy obtainable, the partly unknown expansion rates and internal velocity patterns of these younger stellar groups actually limit the accuracy in their radial-velocity determinations. For more data on these clusters, see Table 4 in Paper I.

\section{Exploitation of the moving-cluster method}

\subsection{Basic cluster model}

The mathematical procedure described in Paper II yields maximum-likelihood estimates for the space velocity of the cluster centroid $\widehat{\boldsymbol{v}}_{0}$, for the internal velocity dispersion of stars within the cluster $\widehat{\sigma}_{v}$, and for the kinematically improved parallax of each star $\widehat{\pi}_{i}, i=1,2, \ldots n$. (We reserve index 0 for the centroid. The caret ${ }^{\wedge}$ signifies an estimated value.) Although additional model parameters could be included, e.g. to describe a possible rotation or non-isotropic dilation of the cluster, the present studies are restricted to the "basic cluster model" in which no such systematic velocity patterns are assumed. In Paper II it was shown, through Monte Carlo simulations of the Hipparcos observations of the Hyades cluster, that the presence of any reasonable amount of rotation and shear in the actual cluster will not significantly bias the solution for the centroid velocity, even though the analysis is restricted to the basic model. One important exception concerns the possible isotropic expansions of gravitationally unbound associations. These could indeed introduce significant biases, which are discussed separately in Sect. 5.7.

\subsection{Observational data}

The Hipparcos Catalogue (ESA 1997) provided input data for each star $i$ in the form of positions in barycentric right ascension $\alpha_{i}$, declination $\delta_{i}$, trigonometric parallax $\widetilde{\pi}_{i}$, the proper-motion components $\widetilde{\mu}_{\alpha i}$ and $\widetilde{\mu}_{\delta i}$, and standard deviations and correlation coefficients for the latter three. (The tilde $\sim$ signifies an observed value, the uncertainty of which needs to be taken into account in the estimation procedure.) The positions and proper motions in the Hipparcos Catalogue are referred to the barycentric ICRS reference system, and consequently all resulting velocities are also in that system.

For each cluster or association, an initial sample of probable member stars was identified from the literature, mainly from studies based on Hipparcos data. However, since the mathematical formalism for obtaining radial velocities is strictly applicable only to stars sharing the same average velocity vector (with a random spread about that value), cluster non-members and binary stars in nonmodelled orbits must be removed from the sample as far as possible. This was done using an iterative rejection procedure described in Paper II. Monte Carlo simulations (Sect. 4.2 in that paper) showed that this procedure works best when adopting the goodness-of-fit rejection limit $g_{\mathrm{lim}}=15$. As illustrated there for the Hyades, this gave the lowest scatter in the centroid radial velocity, as well as in other quality indicators. Therefore, unless otherwise stated, all samples discussed here were cleaned according to this criterion.

Solutions were primarily obtained using data from the main Hipparcos catalogue but, for some clusters, we used also data from the Tycho-2 catalogue (Høg et al. 2000). 
Table 1. Estimated space-velocity components and internal velocity dispersions of clusters and associations analyzed with the moving-cluster method, using astrometric data from the Hipparcos main catalogue. $n_{\text {acc }}$ is the number of stars retained in the cleaned sample for each cluster, $n_{\text {rej }}$ is the number of stars removed in the cleaning process; $\widehat{v}_{0 x}$, $\widehat{v}_{0 y}$, and $\widehat{v}_{0 z}$ are the equatorial (ICRS) components of the estimated space velocity of the cluster centroid; $\widehat{\sigma}_{\mathrm{v}}$ is the estimated internal velocity dispersion among individual stars, calculated as described in Paper II, Appendix A.4. The last three columns give the equatorial coordinates $\left(\alpha_{0}, \delta_{0}\right)$ [deg] of the adopted centroid of the cluster (or, in the case of Ursa Major, its core) and the space velocity component $\widehat{v}_{0 r}$ toward that direction, i.e. an approximate radial velocity of the cluster as a whole. Uncertainties are given as \pm 1 standard error. All velocities are in $\mathrm{km} \mathrm{s}^{-1}$. The electronic version of the table contains, additionally, the six equatorial components of the formal covariance matrix $\operatorname{Cov}\left(\widehat{(}_{0}\right)$, the spherical equatorial coordinates of the convergence point with standard errors, and the total velocity with its standard error.

\begin{tabular}{|c|c|c|c|c|c|c|c|c|c|}
\hline Name & $n_{\text {acc }}$ & $n_{\mathrm{rej}}$ & $\widehat{v}_{0 x}$ & $\widehat{v}_{0 y}$ & $\widehat{v}_{0 z}$ & $\widehat{\sigma}_{\mathrm{v}}$ & $\alpha_{0}$ & $\delta_{0}$ & $\widehat{v}_{0 r}$ \\
\hline Ursa Major & 77 & 4 & $+8.44 \pm 0.41$ & $-12.19 \pm 0.39$ & $-10.16 \pm 0.43$ & $2.82 \pm 0.23$ & 187.3 & +56.4 & $-12.24 \pm 0.46$ \\
\hline Hyades & 168 & 29 & $-5.90 \pm 0.13$ & $+45.65 \pm 0.34$ & $+5.56 \pm 0.10$ & $0.49 \pm 0.04$ & 66.5 & +16.9 & $+39.42 \pm 0.36$ \\
\hline Coma Berenices & 40 & 0 & $-0.82 \pm 0.96$ & $+4.57 \pm 0.15$ & $-4.11 \pm 0.48$ & $0.47 \pm 0.09$ & 187.5 & +26.4 & $-1.64 \pm 1.07$ \\
\hline Pleiades & 60 & 0 & $+1.99 \pm 2.20$ & $+22.95 \pm 3.34$ & $-18.73 \pm 1.82$ & $0.50 \pm 0.13$ & 56.4 & +24.0 & $+10.85 \pm 4.36$ \\
\hline Praesepe & 24 & 0 & $-1.46 \pm 9.03$ & $+48.1 \pm 10.9$ & $+2.00 \pm 5.02$ & $0.67 \pm 0.23$ & 130.2 & +19.6 & $+36.2 \pm 15.0$ \\
\hline Lower Cen Crux & 179 & 1 & $-0.94 \pm 0.29$ & $+18.36 \pm 0.15$ & $-8.59 \pm 0.46$ & $1.13 \pm 0.07$ & 189.6 & -56.2 & $+5.95 \pm 0.53$ \\
\hline Upper Cen Lupus & 218 & 3 & $-4.01 \pm 0.27$ & $+16.47 \pm 0.32$ & $-12.91 \pm 0.37$ & $1.23 \pm 0.08$ & 230.3 & -41.6 & $+1.01 \pm 0.51$ \\
\hline Upper Scorpius & 120 & 0 & $-3.73 \pm 0.56$ & $+9.36 \pm 1.09$ & $-14.57 \pm 0.57$ & $1.33 \pm 0.12$ & 243.4 & -24.1 & $-0.17 \pm 1.33$ \\
\hline Sco OB2 & 510 & 11 & $-1.72 \pm 0.15$ & $+18.19 \pm 0.15$ & $-10.43 \pm 0.21$ & $1.52 \pm 0.06$ & 225.1 & -43.9 & $-1.17 \pm 0.26$ \\
\hline$\alpha$ Per (Per OB3) & 78 & 1 & $-3.23 \pm 0.89$ & $+27.15 \pm 1.26$ & $-11.76 \pm 1.64$ & $0.71 \pm 0.13$ & 52.9 & +47.8 & $+4.53 \pm 2.18$ \\
\hline "HIP 98321" & 59 & 0 & $-3.45 \pm 0.66$ & $+15.55 \pm 1.26$ & $-12.27 \pm 1.13$ & $2.56 \pm 0.26$ & 297.5 & +39.4 & $-19.68 \pm 1.74$ \\
\hline
\end{tabular}

The accuracies in the latter are generally somewhat worse, but since its proper motions incorporate about a century of ground-based observations, the segregation of longperiod binaries may be improved.

\subsection{Clusters and associations studied}

Table 4 in Paper I lists some 15 clusters and associations, for which Hipparcos-type accuracies could potentially yield viable astrometric radial velocities, i.e. with standard errors less than a few $\mathrm{km} \mathrm{s}^{-1}$. In practice the resulting accuracies depend on several additional factors, not considered in that survey, such as the number of member stars actually observed by Hipparcos, the position of the cluster on the sky, the statistical correlations among the astrometric data, and the procedures used to obtain a clean sample. The simplest way to find out whether our method "works" on a particular group of stars is to make a trial solution. We have done that for all the potentially interesting clusters and associations, based on the list in Paper I supplemented with data from the compilations in the Hipparcos Input Catalogue (Turon et al. 1992), by Robichon et al. (1999), and by de Zeeuw et al. (1999).

The original criterion for including a cluster or association in the present study was that it yielded a valid solution with the basic cluster model (Sect. 3.1), including a non-zero estimate of the velocity dispersion. This turned out to be the case for four clusters (Ursa Major, Hyades, Coma Berenices, and Praesepe) and four associations (Lower Centaurus Crux, Upper Centaurus Lupus, Upper Scorpius, and "HIP 98321"). For two more, the Pleiades cluster and the $\alpha$ Persei association, reasonable solutions could be obtained by assuming zero velocity dispersion in the maximum-likelihood procedure (note that the dispersion could still be estimated from the proper-motion residuals, as explained in Sect. 3.4). Considering the astrophysical importance of these clusters, they were therefore included in the study. A separate solution was also made for the Sco OB2 complex (Sect. 5.4).

\subsection{Mathematical bias and noise in the solutions}

Resulting space velocities and internal velocity dispersions for the 11 clusters and associations are in Table 1. $\widehat{v}_{0 x}$, $\widehat{v}_{0 y}$, and $\widehat{v}_{0 z}$ are the equatorial components (ICRS coordinates) of the estimated space velocity of the cluster centroid, while $\widehat{\sigma}_{\mathrm{v}}$ is the velocity dispersion in each coordinate, i.e. the standard deviation of peculiar velocities along a single axis.

The maximum-likelihood estimation tends to underestimate the velocity dispersion, as examined through Monte Carlo simulations in Paper II. In Appendix A.4 of that paper we gave an alternative procedure to estimate the velocity dispersion from the proper-motion residuals perpendicular to the centroid velocity projected on the sky. This was shown to give nearly unbiased results. The velocity dispersions $\widehat{\sigma}_{\mathrm{v}}$ in Table 1 and elsewhere in this paper have therefore been estimated through this alternative procedure.

As was also described in Paper II, the radial-velocity errors among individual stars in the same cluster are not statistically independent, but may carry a significant positive correlation. For each star, the error contains a [nearly constant] component being the uncertainty in the cluster velocity as a whole, plus a random component corresponding to the physical velocity dispersion among 
the individual stars. Averaging over many stars in a given cluster averages away the influence of the velocity dispersion, but has only little effect on the error in the radial velocity of the cluster centroid. This quantity, discussed already in Paper I (e.g. its Table 4), is therefore a limiting accuracy in the average astrometric radial velocity of stars in any one cluster. For certain applications, effects of this noise can be lessened by averaging over different clusters (whose errors are not correlated), e.g., when searching for systematic differences between astrometric and spectroscopic radial-velocity values.

It should be noted that the selection process used to arrive at the final sample may have a significant impact on the estimated internal velocity dispersion. The cleaning process successively removes those stars that deviate most from the mean cluster velocity, thus successively reducing $\widehat{\sigma}_{v}$ for the "cleaner" samples. While designed to remove non-members and other outliers, this procedure naturally affects also the mean characteristics of the remaining stars. For example, in the case of the Hyades, stars in the outskirts of the cluster are preferentially removed during the rejection procedure, meaning that the resulting clean samples more or less correspond to the stars within the tidal radius. In the case of the OB associations, we obtain velocity dispersions of about $1 \mathrm{~km} \mathrm{~s}^{-1}$, a factor of two lower than the estimates for the Orion Nebula Cluster (Jones \& Walker 1988; Tian et al. 1996) and other nearby associations (Mathieu 1986). Thus, although we believe that the velocity dispersions reported here correctly characterize the retained samples, they are not necessarily representative for the cluster or association as a whole.

\subsection{Calculation of astrometric radial velocities}

\subsubsection{The stringent definition of 'radial velocity'}

Recognizing the potential of astrometric radial velocities determined without spectroscopy, a resolution for their stringent definition was adopted at the General Assembly of the International Astronomical Union held in 2000. This resolution (Rickman 2001) defines the geometric concept of radial velocity as $v_{\mathrm{r}}=\mathrm{d} b / \mathrm{d} t_{\mathrm{B}}$, where $b$ is the barycentric coordinate distance to the object and $t_{\mathrm{B}}$ the barycentric coordinate time (TCB) for light arrival at the solar system barycentre. This definition is analogous to the conventional understanding of proper motion as the rate of change in barycentric direction with respect to the time of light reception at the solar-system barycentre.

In this work, we follow this IAU definition of "astrometric radial velocity". The difference with respect to alternative possible definitions is on the order of $v_{\mathrm{r}}^{2} / c$, with $c=$ speed of light (Lindegren et al. 1999; Lindegren \& Dravins, in preparation). Most population I objects (including all clusters and associations considered in this paper) have low velocities, $\left|v_{\mathrm{r}}\right|<50 \mathrm{~km} \mathrm{~s}^{-1}$, resulting in only very small differences, $<10 \mathrm{~m} \mathrm{~s}^{-1}$, between possible alternative definitions.

\subsubsection{Radial velocities for individual stars}

In the basic cluster model, the estimated radial velocity of an individual star is given by

$\widehat{v}_{\mathrm{r} i}=\boldsymbol{r}_{i}^{\prime} \widehat{\boldsymbol{v}}_{0}$

where $\boldsymbol{r}_{i}$ is the unit vector towards star $i$ and $\widehat{\boldsymbol{v}}_{0}$ is the estimated space velocity of the cluster as a whole (actually of its centroid). The prime $\left({ }^{\prime}\right)$ denotes the transpose of the vector. In terms of the equatorial coordinates $\left(\alpha_{i}, \delta_{i}\right)$ of the star we have

$\widehat{v}_{\mathrm{r} i}=\widehat{v}_{0 x} \cos \delta_{i} \cos \alpha_{i}+\widehat{v}_{0 y} \cos \delta_{i} \sin \alpha_{i}+\widehat{v}_{0 z} \sin \delta_{i}$,

where $\left(\widehat{v}_{0 x}, \widehat{v}_{0 y}, \widehat{v}_{0 z}\right)$ are the equatorial velocity components as listed in Table 1 (or as determined by other means). We emphasize that Eq. (2) applies to any star that shares the cluster motion, irrespective of whether that star was present in the database used to determine the cluster motion in the first place.

The standard error $\epsilon\left(\widehat{v}_{\mathrm{r} i}\right)$ of the individual radial velocity is computed from

$\epsilon\left(\widehat{v}_{\mathrm{r} i}\right)^{2}=\boldsymbol{r}_{i}^{\prime} \operatorname{Cov}\left(\widehat{\boldsymbol{v}}_{0}\right) \boldsymbol{r}_{i}+\widehat{\sigma}_{v}^{2}$,

where $\operatorname{Cov}\left(\widehat{\boldsymbol{v}}_{0}\right)$ is the $3 \times 3$ submatrix in $\operatorname{Cov}(\widehat{\boldsymbol{\theta}})$ of all model parameters, referring to the centroid velocity (cf. Eq. (A18) in Paper II). The first term in Eq. (3) represents the uncertainty in the radial component of the common cluster motion, while the second represents the contribution due to the star's peculiar motion.

The complete covariance matrix $\operatorname{Cov}\left(\widehat{\boldsymbol{v}}_{0}\right)$ is only given in the electronic (extended) version of Table 1 . The printed Table 1 gives (following the \pm symbol) the standard errors $\epsilon\left(\widehat{v}_{0 x}\right)$ etc. of the vector components; these equal the square roots of the diagonal elements in $\operatorname{Cov}\left(\widehat{\boldsymbol{v}}_{0}\right)$. Also given for each cluster is the standard error of the radial component $\widehat{v}_{0 \mathrm{r}}=\boldsymbol{r}_{0}^{\prime} \widehat{\boldsymbol{v}}_{0}$ of the centroid motion. This was computed from

$\epsilon\left(\widehat{v}_{0 \mathrm{r}}\right)^{2}=\boldsymbol{r}_{0}^{\prime} \operatorname{Cov}\left(\widehat{\boldsymbol{v}}_{0}\right) \boldsymbol{r}_{0}$,

where $\boldsymbol{r}_{0}$ is the unit vector towards the adopted centroid position $\left(\alpha_{0}, \delta_{0}\right)$ specified in the table. $\widehat{v}_{0 \mathrm{r}}$ can be regarded as an average radial velocity for the cluster as a whole, and its standard error (squared) can be regarded as a typical value for the first term in Eq. (3). Thus, for any star not too far from the cluster centroid, the total standard error of its astrometric radial velocity can be approximately computed as $\left[\epsilon\left(\widehat{v}_{0 \mathrm{r}}\right)^{2}+\widehat{\sigma}_{\mathrm{v}}^{2}\right]^{1 / 2}$, using only quantities from the printed Table 1 .

\subsection{Kinematically improved parallaxes}

Our maximum-likelihood estimation of the cluster space motions also produces estimates of the distances to all individual member stars. A by-product of this movingcluster method is therefore that individual stellar distances are improved, sometimes considerably, compared 
with the original trigonometric determinations. This improvement results from a combination of the trigonometric parallax $\pi_{\text {trig }}$ with the kinematic (secular) parallax $\pi_{\text {kin }}=A \mu / v_{\mathrm{t}}$ derived from the star's proper motion $\mu$ (scaled with the astronomical unit $A$ ) and tangential velocity $v_{\mathrm{t}}$, the latter obtained from the estimated space velocity vector of the cluster. The calculation of secular parallaxes and kinematic distances to stars in moving clusters is of course a classical procedure; what makes our "kinematically improved parallaxes" different from previous methods is that the values are derived without any recourse to spectroscopic data (for details, see Papers I and II).

De Bruijne (1999b) applied the present method to the Scorpius OB2 complex in order to study its HR diagram by means of the improved distances. His "secular parallaxes" are essentially the same as our "kinematically improved parallaxes", being based on the same original formulation by Dravins et al. (1997). The main differences are in the choice of rejection criteria (de Bruijne uses $g_{\text {lim }}=9$ versus our 15 ) and in the practical implementation of the solution (downhill simplex versus our use of analytic derivatives). De Bruijne also made extensive Monte Carlo simulations which demonstrated that the distance estimates are robust against all systematic effects considered, including cluster expansion. For the accuracy of the secular parallaxes, de Bruijne (1999b) used a firstorder formula (his Eqs. (16) and (17)) which explicitly includes a contribution from the (assumed) internal velocity dispersion, but neglects the contribution from the trigonometric parallax error (cf. Eq. (11) in Paper I). By contrast, our error estimates are derived directly from the maximum-likelihood solution (Paper II, Appendix A.3), which in principle takes into account all modelled error sources but in practice underestimates the total error as discussed below. As a result, our error estimates are somewhat smaller than those given by de Bruijne (1999b).

The standard errors for the estimated parallaxes given in this paper are the nominal ones obtained from the maximum-likelihood estimation, which could be an underestimation of the actual errors. Determination of realistic error estimates would require extensive Monte-Carlo experiments based on a detailed knowledge of the actual configuration of stars, their kinematic distributions, etc. This information is in practice unavailable except in idealised simulations, and we therefore choose not to introduce any ad hoc corrections for this. As an example of the possible magnitude of the effect, the Hyades simulations in Paper II could be mentioned: in that particular case, the nominal standard errors required a correction by a factor 1.25 to 1.28 in order to agree with the standard deviations in the actual sample.

\section{Radial velocities for stars in open clusters}

Partial data for astrometric radial velocities and kinematically improved parallaxes of individual stars in more nearby clusters, obtained from Hipparcos data, are in
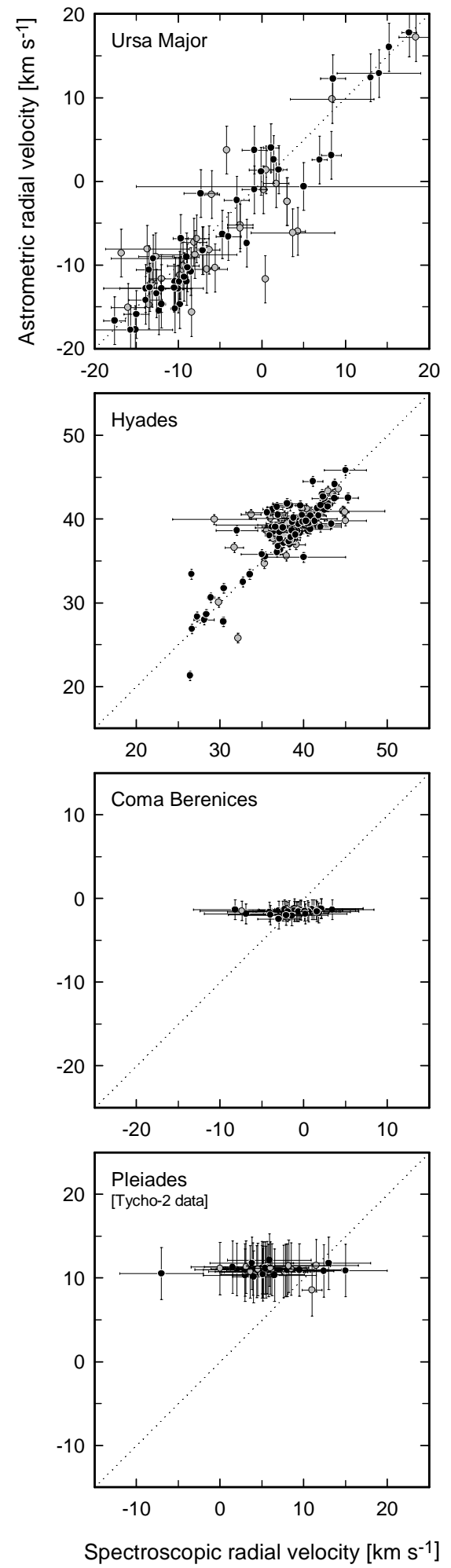

Fig. 3. Astrometrically determined radial velocities compared with spectroscopic values from the literature, for stars in four open clusters. The diagonal lines follow the expected relation $v_{\mathrm{r}}($ astrom $) \simeq v_{\mathrm{r}}$ (spectr). Black symbols denote single stars while certain or suspected binaries are in grey. The top three frames are kinematic solutions obtained from Hipparcos data only. In some cases, including the Pleiades (bottom), somewhat better accuracies are reached using data from the Tycho-2 catalogue, which incorporates almost a century of proper-motion data. 
Table 2. However, the complete listing for the more than 1000 stars in all clusters and associations (some with also Tycho- 2 solutions), is only in the electronic Table 2 .

For some clusters, Fig. 3 shows a comparison between spectroscopic radial velocities compiled from the literature and our currently determined astrometric values. In all cases (also the ones not shown here), the astrometric values agree with the spectroscopic ones within the error limits, verifying the consistency of our method. For several stars (especially rapidly rotating early-type ones with smeared-out spectral lines), the astrometric accuracies are actually substantially better than what has been possible to obtain from spectroscopy. The high accuracies realized for the Hyades enable more detailed comparisons (Sect. 4.2.1).

A subset of known or suspected binary stars for which Hipparcos measurements could be perturbed are defined as stars that are either visual binaries with magnitude difference $\Delta m<4$ mag and a separation $\rho<20$ arcsec according to HIC, the Hipparcos Input Catalogue (Turon et al. 1992); known spectroscopic binaries; or flagged as suspected ones (identified in the Hipparcos Catalogue as a solution of type component, acceleration, orbital, variability-induced mover, or stochastic). In Fig. 3 and later, these stars are plotted in gray.

For finite astrometric accuracy, distant clusters of small angular extent basically yield a single astrometric velocity for all stars. This effect is seen in Fig. 3 for Coma Berenices and the Pleiades, and is similar also for Praesepe and the "HIP 98321" clusters (not plotted).

\subsection{The Ursa Major cluster}

The initial sample consisted of 81 stars, being the sum of the compilations from Soderblom \& Mayor (1993), Dravins et al. (1997) and Montes (2000). A few of the stars identified in a previous kinematic search (Dravins et al. 1997) lacked spectroscopic radial velocities from the literature. Spectroscopic observations of these stars by Gullberg \& Dravins (private comm.) made with the ELODIE spectrometer at Observatoire de Haute-Provence confirmed three of them as probable members. The rejection procedure removed four stars, producing a final sample of 77 .

We chose to include stars not only from the core but also from the extended halo (the moving group sometimes called the Sirius stream), in order to improve the statistical weight of the solution. Not surprisingly, this led to a relatively high velocity dispersion among individual stars. Ursa Major may thus be viewed as a dissolved cluster moving under influence of the Galactic gravity field. Such a fate may be normal for looser open clusters reaching the age (300 Myr) of Ursa Major (Soderblom \& Mayor 1993). For convenience we use the designation "cluster" for the entire sample of stars.

One could imagine that moving groups like Ursa Major could be ideal targets for the method since they have great angular extents. Unfortunately, as Ursa Major illustrates, the high velocity dispersion causes the errors of the estimated astrometric radial velocities to be large. However, it should be noted that the core stars have a much lower velocity dispersion. For instance, we get $\sigma_{\mathrm{v}}=1.05 \pm 0.22 \mathrm{~km} \mathrm{~s}^{-1}$ for the 13 core (nucleus) stars defined by Soderblom \& Mayor (1993). Wielen (1978) considered six core stars with very well determined proper motions and found the velocity dispersion to be on the order of $0.1 \mathrm{~km} \mathrm{~s}^{-1}$ (we get $0.09 \pm 0.03 \mathrm{~km} \mathrm{~s}^{-1}$ for the same six stars), adding that the much larger stream, or moving group, has a dispersion of $\sim 3 \mathrm{~km} \mathrm{~s}^{-1}$, in good agreement with our value. Since we assume this larger dispersion for all the stars, the standard errors are probably overestimated for the core stars.

In the case of Ursa Major, no real gain results from the kinematically improved parallaxes, simply because the relative accuracy in the Hipparcos parallax values is already very good, given the proximity of this cluster.

The spectroscopic velocity values used for Fig. 3 were taken from Soderblom \& Mayor (1993), Duflot et al. (1995), and in a few cases, also the ELODIE observations by Gullberg \& Dravins (private comm.). In the case of Duflot et al., no explicit error is quoted, but rather a flag which seems to correspond to a numerical value that is used in the Hipparcos Input Catalogue: those values were adopted here.

\subsection{The Hyades cluster}

The Hyades cluster (Melotte 25) is the classic example of a moving cluster. Its kinematic distance, derived from a combination of proper motions and spectroscopic radial velocities, has been one of the fundamental starting points for the calibration of the photometric distance scale (e.g. Hanson 1975; Gunn et al. 1988; Schwan 1991, and references therein). Of course, the recent availability of accurate trigonometric parallaxes has now superseded this method for distance determination.

The first detailed study of the distance, structure, membership, dynamics and age of the Hyades cluster, using Hipparcos data, was by Perryman et al. (1998). From a combination of astrometric and spectroscopic radial velocity data, using 180 stars within a radius of $20 \mathrm{pc}$, they derived the space velocity for the cluster centroid, $\boldsymbol{v}_{0}=(-6.32,+45.24,+5.30) \mathrm{km} \mathrm{s}^{-1}$. They also estimated that the true internal velocity dispersion, near the centre of the cluster, is in the range 0.2 to $0.3 \mathrm{~km} \mathrm{~s}^{-1}$.

Our initial sample of stars was selected from the final membership assigned by Perryman et al. (1998), viz. 197 stars classified as probable members $(S=1$ in their Table 2): this equals our sample Hy0 in Paper II.

In earlier kinematic studies of the Hyades, systematic errors in the proper motions have been of major concern, and the probable cause of discrepant distance estimates. In our application, the solution is also sensitive to such errors, but we expect that the high internal consistency of the 
Hipparcos proper motion system, and its accurate linking to the (inertial) extragalactic reference frame (Kovalevsky et al. 1997), have effectively eliminated that problem.

Using the methods described in Paper II, the astrometric radial velocity and its standard deviation for each individual star is obtained through Eqs. (1) and (3), even for stars not retained in the final sample. Individual parallaxes follow directly from the estimation procedure, see Table 2.

The analysis was repeated using proper motions from Tycho-2 instead of the Hipparcos Catalogue. Being based on observations covering a much longer time span, the Tycho-2 data are expected to be more precise for binaries with periods from a few years to $\sim 100$ years. By coincidence, the procedure of cleaning the sample rejected the same number of stars as in the Hipparcos case, albeit different ones (electronic Table 2). Compared to the Hipparcos solution $\left(0.49 \pm 0.04 \mathrm{~km} \mathrm{~s}^{-1}\right)$, we get a smaller velocity dispersion using Tycho-2 $\left(0.34 \pm 0.03 \mathrm{~km} \mathrm{~s}^{-1}\right)$. It can be noted that Makarov et al. (2000) investigated this dispersion in the Hyades as a test of the Tycho- 2 proper motions, finding a dispersion close to our latter value. The two solutions for the cluster centroid velocity are equal to within their uncertainties. However, in the radial direction there is a systematic difference of $\simeq 0.9 \mathrm{~km} \mathrm{~s}^{-1}$, in the sense that the astrometric radial-velocity values from Tycho-2 are smaller than those from the Hipparcos Catalogue (while the kinematically improved parallaxes from Tycho-2 place the stars at slightly greater distances). We have no obvious explanation for this shift, which has analogues for other clusters when comparing Hipparcos and Tycho-2 solutions. Possibly, it reflects the influence of subtle systematic effects in the proper-motion data, which border on the measurement precision. If this is the case, greater confidence should be put on the solution based on the Hipparcos data, as the Tycho-2 system of proper motions was effectively calibrated onto the Hipparcos system. Future space astrometry missions should be able to clarify these matters.

It follows from Eq. (1) that any possible bias in the estimated space velocity $\widehat{\boldsymbol{v}}_{0}$ has only a small influence on the relative astrometric radial velocities in a given cluster, if its angular extent is not too large. Thus the astrophysical differences would still show up as systematic trends when the astrometric radial velocities are compared with spectroscopic values. In such a comparison, the bias in space velocity would mainly introduce a displacement of the zero-point, as mentioned above.

\subsubsection{Hyades: Comparison with spectroscopic data}

The astrophysical potential of astrometric radial velocities begins to appear when the accuracy is sufficiently high to detect differences relative to spectroscopic values (e.g., Dravins et al. 1999a; Dravins 2001, and references therein). Such differences are expected due to stellar surface convection ("granulation"): most photons from
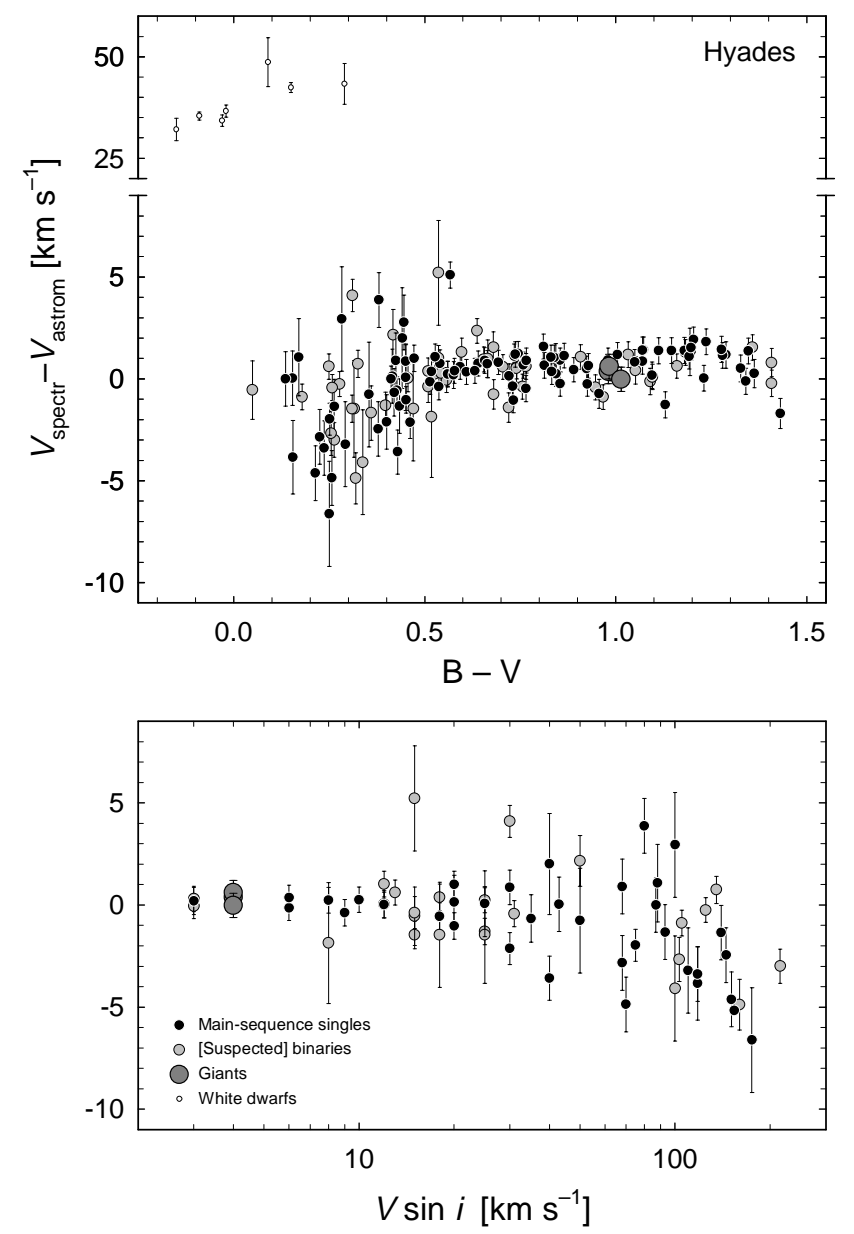

Fig. 4. The Hyades: Differences between spectroscopic radialvelocity values from the literature, and current astrometric determinations. Systematic differences depend on spectral type, and on the projected stellar rotational velocity $V \sin i$. (These dependences are correlated since rapid rotation dominates for early-type stars.) An increased blueshift of spectral lines in stars somewhat hotter than the $\operatorname{Sun}(B-V \simeq 0.3-0.5)$ is theoretically expected due to their more vigorous surface convection, causing greater convective blueshifts. Gravitational redshifts of white-dwarf spectra place them far off main-sequence stars. The error bars show the combined spectroscopic and astrometric errors; stars with errors $>3 \mathrm{~km} \mathrm{~s}^{-1}$ are omitted (except for white dwarfs), as are stars that were not retained by kinematic solutions from both Hipparcos and Tycho-2 data.

a stellar surface are emitted by hot and rising (thus locally blueshifted) convective elements, which contribute a greater number of photons than the cool, dark and sinking areas. The resulting statistical bias causes a convective blueshift, theoretically expected to range from some $0.2 \mathrm{~km} \mathrm{~s}^{-1}$ in red dwarfs, $\simeq 0.4 \mathrm{~km} \mathrm{~s}^{-1}$ in the Sun, to $1.0 \mathrm{~km} \mathrm{~s}^{-1}$ in F-type stars with their more vigorous surface convection, the precise amount varying among different spectral lines with dissimilar conditions of formation. Gravitational redshifts are expected to vary greatly between giants $\left(<0.1 \mathrm{~km} \mathrm{~s}^{-1}\right)$, main-sequence stars $(0.5-$ $1 \mathrm{~km} \mathrm{~s}^{-1}$ ), and white dwarfs (perhaps $30 \mathrm{~km} \mathrm{~s}^{-1}$ ), 
with almost identical redshifts throughout the spectra. Additional effects enter for pulsating stars, stars with expanding atmospheres, and such with other spectral complexities.

Our current accuracies permit such studies to be made for the Hyades, and perhaps marginally for a few other clusters. In Fig. 4 we show the difference between astrometric radial velocities and spectroscopic measurements from the literature. The latter values are taken from the compilation by Perryman et al. (1998), which mostly are measurements by Griffin et al. (1988). The values used here are their original measurements, i.e. not applying any zero-point or other spectral-type dependent "corrections" (such as were later applied in a convergent-point analysis for the Hyades from the same data by Gunn et al. 1988). The plot also includes white dwarfs, whose astrometric velocities are from Eq. (1), and where the spectroscopic data are "weighted values" ( $\mathrm{H} \alpha$ weighted with twice the weight of $\mathrm{H} \beta$ ) from Reid (1996).

The errors for Fig. 4 were calculated as the quadratic sum of the spectroscopic and astrometric uncertainties, where Eq. (3) was used for the latter. Most of the errors are due to the spectroscopic measurements, and it can be noted how the scatter is greater for the [suspected] binary stars.

Over much of the main sequence, convective blueshifts and gravitational redshifts partly cancel one another: an increased convective blueshift in hotter stars is partly balanced by an increased gravitational redshift in these more massive stars. Nevertheless, it is theoretically expected that the strong increase in the vigour of surface convection for middle F-stars $(B-V \simeq 0.4)$ should blueshift their spectra by $\simeq 1 \mathrm{~km} \mathrm{~s}^{-1}$ relative to those of later-type $\mathrm{G}$ or K-type stars $(B-V \simeq 1.0)$. For yet earlier-type stars, there do not yet exist any detailed theoretical models in the literature from which the convective shift can be reliably predicted.

We believe the expected effects are visible in Fig. 4. There is clearly a gradient in the relevant spectral range ( $B-V$ between 0.4 and 0.7 ), with roughly the theoretically expected sign and magnitude of the effect. The trend seems to continue towards even earlier types.

This is not the first time spectral-type dependent radial velocities are seen: a trend of increased spectral blueshift in earlier-type stars was already suggested from residuals in the convergent-point solution by Gunn et al. (1988). A difference between the wavelength scale of giants and dwarfs, suggesting differences in gravitational redshift, was noted from velocity histograms of giants and dwarfs, respectively, in the open cluster NGC 3680 by Nordström et al. (1997).

Both of these works raise an important point relating to the sample selection. Spectroscopic velocities are usually important for the determination of membership probabilities, which are therefore in principle affected by systematics of the kind shown in Fig. 4. Spectral shifts should therefore be taken into account, lest they influence the membership determination and hence the final result, including the spectral shifts themselves. Our initial Hyades sample is based on that of Perryman et al. (1998), who used their compilation of spectroscopic radial velocities to compute membership probabilities. Given the relatively large spectroscopic uncertainties for the earlytype stars, this effect probably did not affect the present Hyades sample. However, as long as the mean spectral shifts remain unknown, e.g. as a function of spectral type along the main sequence, it would be necessary to downweight the more precise spectroscopic velocities in order to avoid possible selection effects related to the spectral shifts.

The errors in the spectroscopic velocities in several of the hottest (and often rapidly rotating) stars are large, making conclusions in that part of the diagram difficult. For such stars with often complex spectra and perhaps expanding atmospheres, the concept of spectroscopic radial velocity must be precisely defined, if studies on the sub-km s$~^{-1}$ are to be feasible (cf. Andersen \& Nordström 1983; Griffin et al. 2000).

\subsubsection{Hyades: The Hertzsprung-Russell diagram}

Already the trigonometric parallaxes from Hipparcos yield quite accurate absolute magnitudes, enabling a precise Hertzsprung-Russell diagram to be constructed. Our kinematically improved parallaxes permit this to be carried further, also verifying the working of our mathematical methods.

Figure 5 shows the gradual improvements in the definition of the Hyades main sequence with successively better data. The top frame shows the apparent magnitudes (i.e., effectively placing all stars at the same mean distance); the second frame shows the improvement from Hipparcos having been able to resolve the depth of the cluster; the third frame uses our kinematically improved parallaxes which, especially for the fainter stars, significantly improve the definition of the main sequence. This is further marginally improved by the use of Tycho- 2 data in the bottom frame. The plot only shows those stars that were retained in both the Hipparcos and Tycho-2 solutions, and excludes [suspected] binary stars as defined in Sect. 4 .

Besides permitting searches for fine structure in the HR diagram, this also confirms the validity of the kinematic solution for the radial-velocity determinations: since no photometric information was used in the solution, such an improvement could hardly be possible unless the underlying physical model is sound. With the kinematically improved parallaxes the error in $M_{H p}$ is typically only $\sim 0.03$ mag, much smaller than the symbol size in Fig. 5 .

In addition to the two giants retained in the solutions, a few stars lie off the main sequence. Below it is HIP 10672 at $B-V=0.567$, a single star quite far (some $30 \mathrm{pc}$ ) from the cluster centre; HIP 17962 at $B-V=0.782$, an eclipsing binary containing a hot white dwarf (Nelson \& Young 1970) which causes a displacement towards the blue; and HIP 19862 at $B-V=0.924$, with an uncertain colour 

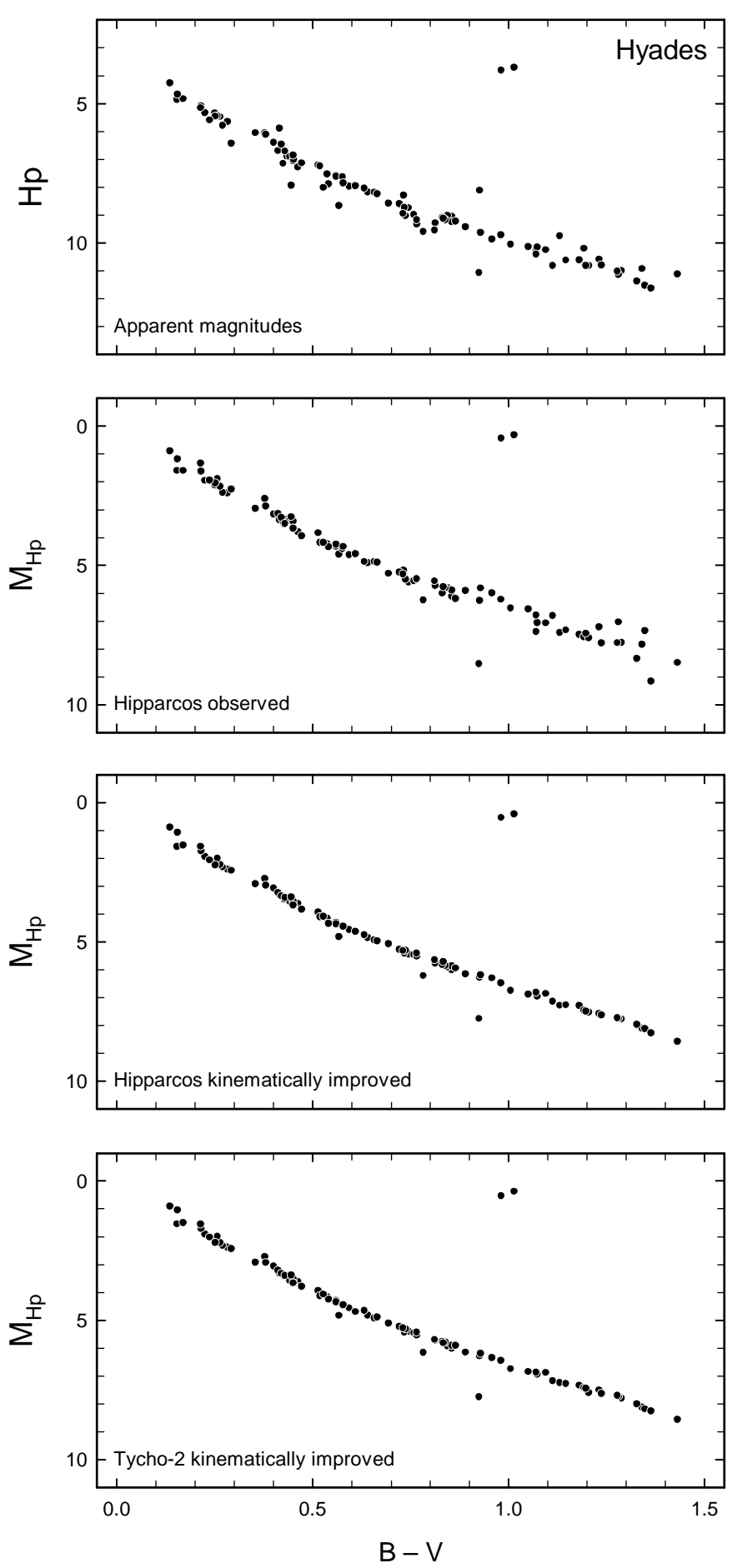

Fig. 5. The Hyades: Improved definition of the HertzsprungRussell diagram from kinematically improved parallaxes. From top: a) apparent magnitudes as measured by Hipparcos; b) absolute magnitudes from Hipparcos parallaxes tighten the main sequence since the cluster depth is resolved; c) absolute magnitudes from kinematically improved parallaxes computed from Hipparcos data greatly improve the definition of the main sequence (especially for the fainter stars), further marginally improved by the use of Tycho-2 data $\mathbf{d}$ ). This permits searches for fine structure in the HR-diagram, and also confirms the validity of the kinematic solution for the radial-velocity determinations. Only single stars, retained by the kinematic solutions from both Hipparcos and Tycho-2 data, are plotted. index in the Hipparcos Catalogue $\left(\sigma_{B-V}=0.301\right)-$ the value $B-V=1.281 \pm 0.009$ given in the Hipparcos Input Catalogue (Turon et al. 1992) would place it exactly on the main sequence. All these stars have an uncertainty in $M_{H p}$ of $0.05 \mathrm{mag}$ or less, meaning that they are not misplaced vertically. Simulations of a Hyades-type cluster by Portegies Zwart et al. (2001) showed that a few stars end up below the main sequence as a result of binary interaction leading to blueward displacements. From Fig. 5 it is difficult to tell where the turnoff point really is: some stars to the far left may be blue stragglers.

The rest of the cluster stars lie practically on a single curve, which can be considered a confirmation of the parallax improvement. It is not clear whether the remaining spread of the main sequence in $M_{H p}$ is real or can be accounted for by uncertainties in $B-V$, although these are small. Effects such as differential reddening within the cluster seem unlikely: Taylor (1980) found only a very small colour excess $E(B-V)=0.003 \pm 0.002 \mathrm{mag}$ for the Hyades.

Improved absolute magnitudes were also determined by de Bruijne et al. (2001) based on our original method (Dravins et al. 1997). Lebreton et al. (2001) compared our kinematically improved parallaxes to those by de Bruijne et al., finding excellent agreement in all values, except for one star (HIP 28356). We note that this particular star is the one located the furthest from the cluster centre, and is also one where our cleaning procedure removed it from the Tycho-2 solution (although it was retained in Hipparcos data). It may be a long-period binary whose photocentric motion causes a deviation in the modulus of the measured proper motion, if not in its direction.

For further discussions of the post-Hipparcos HR diagram for the Hyades, see Perryman et al. (1998), Madsen (1999), Castellani et al. (2001), de Bruijne et al. (2001), and Lebreton (2000), where the latter four have used the improved parallaxes.

\subsection{The Coma Berenices cluster}

The Coma Berenices sample is made up of the 40 Hipparcos stars in Odenkirchen et al. (1998). This sample includes four stars that, while slightly beyond their selected limit for membership, nonetheless were considered to "very probably also belong to the cluster". Since the small number of stars made the solution unstable already after rejecting two of them, the results in Table 1 are given for the full sample $\left(g_{\lim }=\infty\right)$. Although the typical errors in the astrometric radial velocities are only $1.2 \mathrm{~km} \mathrm{~s}^{-1}$, the precision of published spectroscopic values is generally insufficient for meaningful comparisons.

The kinematically improved parallaxes produce only a slight improvement in the HR diagram at the red end of the main sequence (Fig. 6). 


\subsection{The Pleiades cluster}

The sample contains 60 stars from van Leeuwen (1999, and private comm.). The stars are too few and/or the cluster too distant for the basic cluster model to give a direct estimate for the velocity dispersion. It has instead been estimated by the procedure described in Appendix A.4 of Paper II. No improvements to the parallaxes result from the kinematic solution, since our method is unable to resolve the depth of this cluster; it therefore in essence ascribes the same distance to every star. The small angular extent means that also the astrometric radial velocity is practically the same for all stars (Fig. 3, bottom).

As seen in Fig. 6, the Pleiades main sequence occupies a position at the lower edge of the distribution for the different clusters. The Pleiades cluster is at the focus of an ongoing debate concerning possible localized systematic errors in the Hipparcos parallaxes (see e.g. Pinsonneault et al. 1998, 2000; Robichon et al. 1999; Narayanan \& Gould 1999; van Leeuwen 1999, 2000; Paper II; Stello \& Nissen 2001). If such errors were present in our input data, they would not be detected by the present maximumlikelihood method, but would affect also the kinematically improved parallaxes. Consequently, the present results provide no direct new information towards the resolution of this issue.

\subsection{The Praesepe cluster}

The investigated sample was based on 24 stars from van Leeuwen (1999, and private comm.). As for the preceding two clusters, the solution places the stars at practically the same distance. The resulting mean astrometric radial velocity has an error of $\simeq 15 \mathrm{~km} \mathrm{~s}^{-1}$. While demonstrating the applicability of the method, this present accuracy is insufficient for detailed stellar studies.

\subsection{A composite HR diagram}

Kinematically improved parallaxes from the different clusters enable a very detailed comparison between the main sequences of different clusters. Such a Hertzsprung-Russell diagram for five nearby clusters is in Fig. 6. We again stress that, while our kinematic solution reduces the random noise, it does not address any possible systematic effects and therefore cannot decide whether, e.g. the systematic shifts in luminosity between different clusters are caused by astrophysical or by instrumental effects.

However, the very low noise level permits to search for morphological fine structures in the HR diagram. From post-Hipparcos data for the Hyades, de Bruijne et al. (2000, 2001) suggested the existence of two underpopulated main-sequence segments around $B-V$ approx 0.38 and 0.5, identified as "Böhm-Vitense gaps", theoretically predicted due to changing efficiencies of stellar convection at temperatures corresponding to those particular colours. However, these gaps are not seen in other clusters, and their "existence" is consistent with small-number statistics

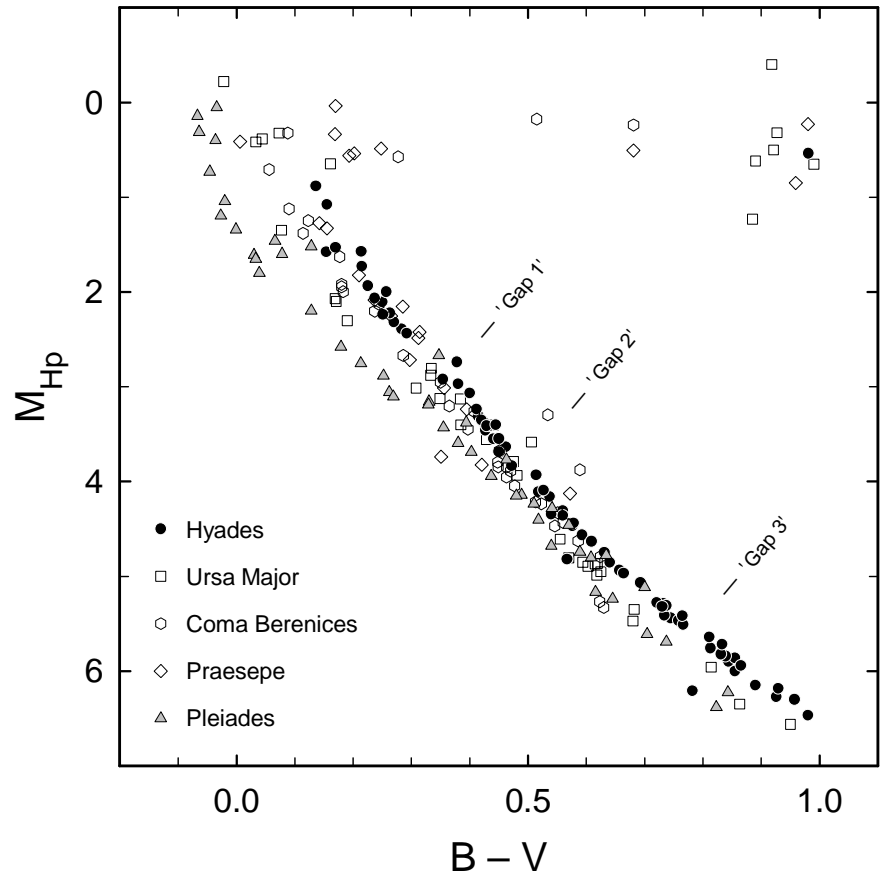

Fig. 6. Hertzsprung-Russell diagram for single stars in the better-defined open clusters, obtained using kinematically improved parallaxes from Hipparcos data. Hp magnitudes are given since these are more precise than ground-based $V$ ones, and since $H p$ values are available for all stars in this sample (values for $M_{H p}$ fall quite close to those of $M_{V}$ ). The random errors in these kinematically improved parallaxes are lower by factors of typically 2 or 3 compared with the original Hipparcos values, and the absolute magnitudes are correspondingly more precise, beginning to reveal fine structures in the HR diagram. For the Hyades, de Bruijne et al. $(2000,2001)$ suggested the existence of two underpopulated main-sequence segments around $B-V \simeq 0.38$ and 0.5 , identified as "Böhm-Vitense gaps", theoretically predicted due to changing efficiencies of stellar convection at the corresponding temperatures. However, these gaps are not seen in other clusters, and their "existence" is consistent with small-number statistics causing random clustering. This probably also applies to the apparent "Gap 3" at $B-V \simeq 0.8$.

causing random clustering. This probably also applies to the apparent "Gap 3" at $B-V \simeq 0.8$. (Of course, the detectability of such gaps depends also on the precision in the other axis, i.e. the colour index.) Although some authors have suggested a possible presence of such "gaps" (e.g., Rachford \& Canterna 2000), extensive analyses of field stars, using Hipparcos parallaxes, failed to show any (Newberg \& Yanny 1998).

A certain fine structure (wiggles, etc.) is theoretically expected in the HR- diagram (e.g., Siess et al. 1997); possible hints of that are becoming visible for the Hyades.

\section{Application to OB associations}

The procedures of determining astrometric radial velocities were applied also to a number of nearby associations of young stars. The situation is here somewhat different 
Table 2. Data for individual stars for the better-defined clusters. Estimated radial velocities and their standard errors are derived from Eqs. (1) and (3), using the adopted solutions in Table 1 and the corresponding covariances. Columns: HIP = Hipparcos Catalogue number, $\widehat{v}_{\mathrm{r}, \mathrm{Hip}}=$ astrometric radial velocity $\left[\mathrm{km} \mathrm{s}^{-1}\right]$ obtained from the kinematic solution using data from the Hipparcos main catalogue; $\widehat{\pi}_{\text {Hip }}=$ estimated parallax [mas] from the kinematic solution using data from the Hipparcos main catalogue; $\epsilon\left(\widehat{\pi}_{\text {Hip }}\right)=$ standard error [mas] of this estimated parallax. Part 1: data for Ursa Major and Hyades. The complete table for all clusters and associations, including results and errors obtained from both Hipparcos and Tycho-2 data, is available in electronic form.

\begin{tabular}{|c|c|c|c|c|c|c|c|c|c|c|c|c|c|c|}
\hline HIP & $\hat{v}_{\mathrm{r}, \mathrm{Hip}}$ & $\hat{\pi}_{\mathrm{Hip}}$ & $\epsilon\left(\hat{\pi}_{\mathrm{Hip}}\right)$ & HIP & $\hat{v}_{\mathrm{r}, \mathrm{Hip}}$ & $\hat{\pi}_{\mathrm{Hip}}$ & $\epsilon\left(\hat{\pi}_{\mathrm{Hip}}\right)$ & HIP & $\hat{v}_{\mathrm{r}, \mathrm{Hip}}$ & $\hat{\pi}_{\mathrm{Hip}}$ & $\epsilon\left(\hat{\pi}_{\mathrm{Hip}}\right)$ & HIP & $\hat{v}_{\mathrm{r}, \mathrm{Hip}}$ & $\epsilon\left(\hat{\pi}_{\mathrm{Hip}}\right)$ \\
\hline
\end{tabular}

\section{Ursa Major}

$\begin{array}{rrrr}2213 & 3.1 & 6.6 & 0.6 \\ 8486 & 3.8 & 42.6 & 3.8 \\ 8497 & 3.7 & 42.4 & 0.9 \\ 10403 & -7.3 & 24.4 & 0.9 \\ 10552 & -1.0 & 28.9 & 2.7 \\ 17874 & 1.4 & 15.8 & 0.6 \\ 18512 & -6.0 & 63.3 & 2.0 \\ 19655 & -12.0 & 38.5 & 1.3 \\ 19855 & -8.3 & 47.8 & 1.1 \\ 19859 & -8.3 & 47.1 & 1.1 \\ 21818 & -11.7 & 74.6 & 1.2 \\ 23875 & -9.0 & 36.7 & 0.8 \\ 25110 & -12.0 & 47.7 & 0.5 \\ 27072 & -6.8 & 111.5 & 0.6 \\ 27913 & -14.8 & 115.4 & 1.1 \\ 28954 & -14.7 & 64.7 & 0.9 \\ 30277 & -5.2 & 13.9 & 0.6 \\ 30630 & -15.6 & 68.3 & 1.1 \\ 32349 & -10.1 & 379.2 & 1.6 \\ 36704 & -17.7 & 50.8 & 1.3 \\ & & & \end{array}$

$\begin{array}{rrrr}38228 & -17.7 & 45.8 & 0.9 \\ 42438 & -15.4 & 70.0 & 0.7 \\ 43352 & -6.8 & 14.0 & 1.1 \\ 46298 & -12.8 & 19.1 & 0.9 \\ 48341 & -12.8 & 16.1 & 0.8 \\ 48356 & -10.6 & 11.6 & 0.8 \\ 49593 & -16.6 & 35.7 & 0.8 \\ 49929 & -15.1 & 15.1 & 0.8 \\ 50335 & -15.9 & 12.4 & 0.8 \\ 51814 & -15.2 & 37.8 & 0.6 \\ 53910 & -14.7 & 41.1 & 0.6 \\ 53985 & -14.2 & 85.7 & 1.4 \\ 55454 & -6.2 & 75.8 & 1.7 \\ 56154 & -7.4 & 15.7 & 0.8 \\ 57283 & -5.6 & 9.4 & 0.7 \\ 57548 & -9.2 & 299.9 & 2.2 \\ 58001 & -13.4 & 39.0 & 0.7 \\ 59496 & -12.7 & 35.3 & 1.2 \\ 59514 & -12.8 & 65.6 & 1.5 \\ 59774 & -12.7 & 40.0 & 0.6\end{array}$

$\begin{array}{rrrr}61100 & -12.2 & 41.1 & 2.9 \\ 61481 & -12.0 & 38.1 & 1.1 \\ 61621 & -1.0 & 29.0 & 0.7 \\ 61946 & -11.8 & 43.0 & 0.8 \\ 62512 & -11.6 & 40.8 & 3.0 \\ 62956 & -11.4 & 40.3 & 0.6 \\ 63008 & -8.8 & 28.3 & 1.5 \\ 63503 & -11.2 & 40.0 & 0.6 \\ 64405 & -9.0 & 11.5 & 1.2 \\ 64532 & -10.8 & 38.9 & 0.7 \\ 65327 & -10.5 & 39.8 & 1.4 \\ 65378 & -10.3 & 41.7 & 0.6 \\ 65477 & -10.3 & 40.2 & 0.6 \\ 66459 & -8.1 & 91.7 & 1.2 \\ 69989 & -2.3 & 38.2 & 0.8 \\ 71876 & -8.2 & 23.5 & 0.6 \\ 72944 & -0.6 & 101.7 & 1.7 \\ 73996 & -1.5 & 50.9 & 0.8 \\ 75312 & -1.6 & 53.8 & 1.2 \\ 76267 & -0.3 & 43.6 & 0.8\end{array}$

$\begin{array}{rrrr}77233 & 2.6 & 21.3 & 0.8 \\ 80337 & 12.4 & 77.6 & 0.9 \\ 80686 & 12.3 & 82.6 & 0.6 \\ 80902 & -8.6 & 36.7 & 0.6 \\ 82780 & 4.0 & 7.2 & 0.6 \\ 83988 & -2.4 & 46.5 & 1.8 \\ 83996 & -2.4 & 46.1 & 2.8 \\ 87079 & -6.3 & 25.1 & 0.6 \\ 88694 & 16.0 & 57.6 & 0.8 \\ 91159 & 9.8 & 28.9 & 1.5 \\ 94083 & -6.6 & 36.6 & 0.5 \\ 96258 & 1.2 & 39.2 & 0.5 \\ 101027 & 17.2 & 33.0 & 0.9 \\ 103738 & 17.7 & 14.5 & 0.8 \\ 106481 & 2.6 & 26.2 & 0.5 \\ 110091 & 12.9 & 24.1 & 0.9 \\ 112460 & 1.4 & 198.2 & 2.0\end{array}$

\section{Hyades}

\begin{tabular}{|c|c|c|c|c|c|c|c|c|c|c|c|c|c|c|c|}
\hline 10672 & 21.30 & 17.0 & 0.3 & 19870 & 37.88 & 20.6 & 0.3 & 20711 & 38.60 & 21.7 & 0.3 & 21474 & 40.54 & 20.6 & 0.3 \\
\hline 12709 & 25.79 & 54.0 & 0.8 & 19877 & 38.51 & 21.5 & 0.3 & 20712 & 38.83 & 20.9 & 0.3 & 21482 & 38.65 & 52.0 & 0.6 \\
\hline 13600 & 27.73 & 15.3 & 0.3 & 19934 & 37.80 & 19.7 & 0.3 & 20741 & 39.50 & 22.2 & 0.4 & 21543 & 40.69 & 19.7 & 0.6 \\
\hline 13806 & 26.86 & 24.5 & 0.3 & 20019 & 38.56 & 21.1 & 0.3 & 20745 & 39.84 & 25.1 & 0.8 & 21589 & 40.94 & 22.3 & 0.4 \\
\hline 13834 & 27.97 & 30.5 & 0.3 & 20056 & 38.46 & 21.9 & 0.3 & 20751 & 39.93 & 23.0 & 0.5 & 21637 & 39.67 & 23.3 & 0.3 \\
\hline 13976 & 28.60 & 42.8 & 0.5 & 20082 & 38.72 & 22.4 & 0.5 & 20762 & 39.83 & 21.3 & 0.5 & 21654 & 40.90 & 22.8 & 0.4 \\
\hline 14976 & 28.32 & 25.0 & 0.3 & 20087 & 38.03 & 18.3 & 0.2 & 20815 & 39.71 & 21.2 & 0.3 & 21670 & 41.17 & 20.4 & 0.4 \\
\hline 15300 & 30.06 & 25.5 & 0.6 & 20130 & 38.34 & 21.9 & 0.3 & 20826 & 39.99 & 22.3 & 0.4 & 21683 & 40.77 & 18.3 & 0.3 \\
\hline 15563 & 31.72 & 32.1 & 0.4 & 20146 & 38.66 & 21.6 & 0.4 & 20827 & 39.82 & 20.5 & 0.4 & 21723 & 41.08 & 22.9 & 0.6 \\
\hline 15720 & 30.60 & 31.0 & 0.5 & 20205 & 38.91 & 22.1 & 0.3 & 20842 & 38.97 & 20.2 & 0.3 & 21741 & 39.75 & 16.6 & 0.3 \\
\hline 16529 & 32.47 & 23.7 & 0.3 & 20215 & 38.84 & 24.3 & 0.4 & 20850 & 39.89 & 21.6 & 0.4 & 21762 & 40.81 & 21.1 & 0.8 \\
\hline 16908 & 33.39 & 21.3 & 0.3 & 20237 & 38.56 & 22.2 & 0.3 & 20889 & 39.39 & 21.9 & 0.3 & 22177 & 41.71 & 22.0 & 0.7 \\
\hline 17766 & 35.51 & 27.3 & 0.5 & 20261 & 39.04 & 21.0 & 0.3 & 20890 & 39.32 & 20.9 & 0.3 & 22203 & 41.44 & 21.2 & 0.4 \\
\hline 17962 & 35.44 & 21.0 & 0.3 & 20284 & 39.17 & 20.6 & 0.3 & 20894 & 39.78 & 22.2 & 0.4 & 22224 & 41.21 & 22.9 & 0.5 \\
\hline 18018 & 34.67 & 24.3 & 0.7 & 20349 & 38.43 & 20.2 & 0.3 & 20899 & 39.65 & 21.6 & 0.3 & 22253 & 40.40 & 18.3 & 0.4 \\
\hline 18170 & 35.77 & 23.7 & 0.3 & 20350 & 38.80 & 21.4 & 0.3 & 20901 & 40.02 & 21.3 & 0.3 & 22265 & 41.21 & 20.0 & 0.4 \\
\hline 18322 & 36.32 & 21.5 & 0.4 & 20357 & 39.20 & 20.4 & 0.3 & 20916 & 39.79 & 18.7 & 0.5 & 22271 & 39.76 & 27.3 & 0.5 \\
\hline 18327 & 36.03 & 24.4 & 0.4 & 20400 & 39.27 & 22.3 & 0.3 & 20935 & 39.67 & 21.8 & 0.3 & 22350 & 40.86 & 20.6 & 0.4 \\
\hline 18658 & 36.94 & 23.5 & 0.5 & 20419 & 39.46 & 22.1 & 0.5 & 20948 & 39.65 & 21.8 & 0.3 & 22380 & 41.27 & 20.9 & 0.4 \\
\hline 18735 & 36.58 & 22.0 & 0.3 & 20440 & 39.27 & 21.7 & 0.5 & 20949 & 38.12 & 17.3 & 0.3 & 22394 & 40.18 & 20.4 & 0.4 \\
\hline 18946 & 36.76 & 21.0 & 0.4 & 20455 & 39.04 & 21.1 & 0.3 & 20951 & 39.65 & 22.3 & 0.4 & 22422 & 41.65 & 20.8 & 0.4 \\
\hline 19148 & 37.44 & 20.9 & 0.3 & 20484 & 39.17 & 20.8 & 0.3 & 21008 & 39.46 & 19.1 & 0.3 & 22550 & 42.15 & 21.3 & 0.4 \\
\hline 19207 & 37.56 & 21.6 & 0.4 & 20485 & 39.27 & 24.9 & 0.5 & 21029 & 39.93 & 21.8 & 0.3 & 22565 & 41.43 & 19.3 & 0.3 \\
\hline 19261 & 37.65 & 22.6 & 0.3 & 20491 & 38.04 & 18.7 & 0.3 & 21036 & 40.15 & 22.4 & 0.3 & 22566 & 41.85 & 16.4 & 0.3 \\
\hline 19263 & 37.53 & 21.7 & 0.4 & 20492 & 39.38 & 21.0 & 0.4 & 21039 & 39.99 & 21.7 & 0.4 & 22654 & 41.48 & 19.2 & 0.5 \\
\hline 19316 & 37.91 & 20.8 & 0.5 & 20527 & 39.46 & 22.6 & 0.6 & 21066 & 40.34 & 21.9 & 0.4 & 22850 & 41.61 & 15.9 & 0.3 \\
\hline 19365 & 35.57 & 14.7 & 0.2 & 20542 & 39.17 & 22.1 & 0.3 & 21099 & 39.51 & 21.7 & 0.4 & 23069 & 42.48 & 18.0 & 0.4 \\
\hline 19441 & 38.15 & 28.3 & 0.5 & 20557 & 38.59 & 23.6 & 0.4 & 21112 & 40.22 & 19.6 & 0.3 & 23214 & 42.44 & 23.3 & 0.4 \\
\hline 19504 & 37.66 & 22.5 & 0.3 & 20563 & 39.12 & 22.3 & 0.5 & 21123 & 39.87 & 22.1 & 0.4 & 23312 & 42.94 & 19.3 & 0.5 \\
\hline 19554 & 38.28 & 26.5 & 0.4 & 20567 & 39.24 & 19.8 & 0.4 & 21137 & 40.09 & 22.8 & 0.3 & 23497 & 41.84 & 19.1 & 0.3 \\
\hline 19591 & 37.03 & 25.2 & 0.4 & 20577 & 39.27 & 21.8 & 0.4 & 21138 & 40.13 & 21.1 & 1.0 & 23498 & 42.90 & 18.4 & 0.5 \\
\hline 19781 & 38.43 & 19.8 & 0.3 & 20605 & 39.40 & 20.8 & 1.5 & 21152 & 40.46 & 23.8 & 0.4 & 23701 & 43.34 & 18.5 & 0.7 \\
\hline 19786 & 38.57 & 21.6 & 0.4 & 20614 & 39.06 & 21.6 & 0.3 & 21179 & 40.37 & 21.9 & 0.6 & 23750 & 42.67 & 18.8 & 0.4 \\
\hline 19789 & 37.50 & 17.6 & 0.2 & 20635 & 38.60 & 21.1 & 0.3 & 21256 & 39.56 & 23.2 & 0.4 & 23983 & 43.56 & 19.0 & 0.4 \\
\hline 19793 & 37.31 & 22.4 & 0.3 & 20641 & 38.62 & 22.5 & 0.3 & 21261 & 39.89 & 21.6 & 0.5 & 24019 & 40.81 & 17.9 & 0.3 \\
\hline 19796 & 38.65 & 22.0 & 0.3 & 20648 & 39.25 & 21.8 & 0.3 & 21267 & 40.49 & 21.8 & 0.4 & 24116 & 42.53 & 12.4 & 0.3 \\
\hline 19808 & 38.58 & 21.9 & 0.5 & 20661 & 39.48 & 21.3 & 0.3 & 21273 & 40.37 & 22.4 & 0.5 & 24923 & 44.17 & 17.7 & 0.5 \\
\hline 19834 & 38.53 & 21.3 & 0.8 & 20679 & 39.27 & 22.9 & 0.5 & 21317 & 40.38 & 22.0 & 0.4 & 26382 & 44.49 & 19.7 & 0.5 \\
\hline 19862 & 38.46 & 21.8 & 0.5 & 20686 & 39.17 & 22.1 & 0.4 & 21459 & 39.43 & 23.6 & 0.3 & 28356 & 45.83 & 13.4 & 0.6 \\
\hline
\end{tabular}

from that of the previously discussed older clusters because (at least some of) these younger associations may be undergoing significant expansion, or have otherwise complex patterns of stellar motion on levels comparable to our desired accuracies. We recall that the present moving-cluster method is based upon measuring the rate of angular expansion or contraction: it cannot therefore segregate whether a change in angular scale occurs because the cluster is approaching or expanding. While - on the accuracy levels aimed at - this should not be a problem for the older clusters, the likely expansion of young associations may introduce significant biases in the solution. 
Table 2. (Continued) Data for individual stars for the better-defined clusters. Part 2: data for Coma Berenices, Pleiades and Praesepe. The complete table for all clusters and associations, including results and errors obtained from both Hipparcos and Tycho-2 data, is available in electronic form.

\begin{tabular}{|c|c|c|c|c|c|c|c|c|c|c|c|c|c|c|c|}
\hline HIP & $\hat{v}_{\mathrm{r}, \mathrm{Hip}}$ & $\hat{\pi}_{\mathrm{Hip}}$ & $\epsilon\left(\hat{\pi}_{\mathrm{Hip}}\right)$ & HIP & $\hat{v}_{\mathrm{r}, \mathrm{Hip}}$ & $\hat{\pi}_{\text {Hip }}$ & $\epsilon\left(\hat{\pi}_{\mathrm{Hip}}\right)$ & HIP & $\hat{v}_{\mathrm{r}, \mathrm{Hip}}$ & $\hat{\pi}_{\mathrm{Hip}}$ & $\epsilon\left(\hat{\pi}_{\mathrm{Hip}}\right)$ & HIP & $\hat{v}_{\mathrm{r}, \mathrm{Hip}}$ & $\hat{\pi}_{\mathrm{Hip}}$ & $\epsilon\left(\hat{\pi}_{\mathrm{Hip}}\right)$ \\
\hline \multicolumn{16}{|c|}{ Coma Berenices } \\
\hline 59364 & -1.4 & 11.0 & 0.6 & 60123 & -1.6 & 11.5 & 0.6 & 60525 & -1.5 & 10.7 & 0.6 & 61205 & -2.2 & 13.2 & 1.0 \\
\hline 59399 & -1.3 & 10.5 & 0.8 & 60206 & -1.4 & 11.3 & 0.7 & 60582 & -1.5 & 10.3 & 0.7 & 61295 & -1.6 & 10.4 & 0.5 \\
\hline 59527 & -1.4 & 11.4 & 0.6 & 60266 & -1.4 & 11.0 & 0.6 & 60611 & -1.3 & 11.4 & 0.7 & 61402 & -1.8 & 11.0 & 0.7 \\
\hline 59833 & -1.3 & 10.4 & 0.6 & 60293 & -1.5 & 10.9 & 0.8 & 60649 & -1.6 & 12.0 & 0.6 & 62384 & -1.7 & 11.9 & 0.7 \\
\hline 59957 & -1.4 & 11.3 & 0.6 & 60304 & -1.6 & 11.1 & 0.7 & 60697 & -1.6 & 13.1 & 0.5 & 62763 & -2.1 & 10.9 & 0.6 \\
\hline 60014 & -1.8 & 12.3 & 0.6 & 60347 & -1.2 & 11.2 & 0.6 & 60746 & -1.6 & 11.6 & 0.5 & 62805 & -2.0 & 12.5 & 0.8 \\
\hline 60025 & -1.2 & 12.2 & 0.7 & 60351 & -1.5 & 11.4 & 0.5 & 60797 & -1.6 & 10.9 & 0.6 & 63493 & -2.0 & 11.7 & 0.7 \\
\hline 60063 & -1.3 & 11.5 & 0.7 & 60406 & -1.5 & 10.6 & 0.7 & 61071 & -1.5 & 11.2 & 0.5 & 64235 & -2.8 & 12.8 & 1.0 \\
\hline 60066 & -1.4 & 12.3 & 0.5 & 60458 & -1.6 & 11.6 & 0.6 & 61074 & -1.7 & 11.9 & 0.6 & 65466 & -2.5 & 11.7 & 0.6 \\
\hline 60087 & -1.2 & 11.6 & 0.5 & 60490 & -1.5 & 11.8 & 0.5 & 61147 & -1.9 & 11.6 & 0.6 & 65508 & -2.6 & 11.2 & 0.8 \\
\hline \multicolumn{16}{|c|}{ Pleiades } \\
\hline 16217 & 9.1 & 8.6 & 0.2 & 17289 & 11.2 & 7.9 & 0.3 & 17573 & 10.7 & 8.5 & 0.2 & 17847 & 11.0 & 8.3 & 0.2 \\
\hline 16407 & 9.6 & 8.7 & 0.2 & 17317 & 11.4 & 8.4 & 0.3 & 17579 & 10.6 & 8.4 & 0.2 & 17851 & 10.9 & 8.7 & 0.2 \\
\hline 16635 & 9.9 & 8.2 & 0.4 & 17325 & 12.4 & 8.7 & 0.2 & 17583 & 10.2 & 8.5 & 0.2 & 17862 & 10.8 & 8.3 & 0.2 \\
\hline 16639 & 10.2 & 8.2 & 0.3 & 17401 & 10.9 & 8.5 & 0.2 & 17588 & 10.6 & 8.3 & 0.2 & 17892 & 11.7 & 8.4 & 0.2 \\
\hline 16753 & 10.9 & 8.7 & 0.3 & 17481 & 12.2 & 8.3 & 0.2 & 17607 & 12.2 & 7.9 & 0.4 & 17900 & 11.2 & 8.2 & 0.2 \\
\hline 16979 & 11.2 & 8.1 & 0.3 & 17489 & 10.7 & 8.3 & 0.2 & 17608 & 10.9 & 8.1 & 0.2 & 17923 & 11.1 & 8.2 & 0.3 \\
\hline 17000 & 11.1 & 8.4 & 0.2 & 17497 & 11.1 & 8.4 & 0.2 & 17625 & 10.1 & 8.4 & 0.2 & 17999 & 11.1 & 8.4 & 0.2 \\
\hline 17020 & 10.3 & 8.1 & 0.3 & 17499 & 10.8 & 8.5 & 0.2 & 17664 & 10.7 & 8.6 & 0.2 & 18050 & 10.9 & 8.5 & 0.2 \\
\hline 17034 & 10.2 & 8.6 & 0.2 & 17511 & 11.7 & 8.1 & 0.3 & 17692 & 11.0 & 8.3 & 0.2 & 18154 & 10.8 & 8.3 & 0.3 \\
\hline 17044 & 10.4 & 8.4 & 0.3 & 17525 & 9.8 & 8.9 & 0.5 & 17694 & 11.4 & 8.5 & 0.3 & 18263 & 11.1 & 9.1 & 0.4 \\
\hline 17091 & 10.9 & 8.8 & 0.3 & 17527 & 10.5 & 8.6 & 0.2 & 17702 & 10.9 & 8.1 & 0.2 & 18266 & 12.4 & 8.3 & 0.4 \\
\hline 17125 & 8.9 & 8.1 & 0.3 & 17531 & 10.6 & 7.9 & 0.2 & 17704 & 10.8 & 8.3 & 0.2 & 18431 & 11.7 & 8.6 & 0.2 \\
\hline 17168 & 8.5 & 7.5 & 0.4 & 17547 & 8.7 & 8.9 & 0.3 & 17729 & 10.3 & 8.6 & 0.2 & 18544 & 12.8 & 9.5 & 0.3 \\
\hline 17225 & 10.9 & 8.6 & 0.3 & 17552 & 12.1 & 9.0 & 0.2 & 17776 & 11.2 & 8.7 & 0.2 & 18559 & 11.3 & 8.2 & 0.2 \\
\hline 17245 & 10.0 & 8.0 & 0.3 & 17572 & 11.2 & 8.5 & 0.2 & 17791 & 10.8 & 8.0 & 0.2 & 18955 & 12.1 & 8.5 & 0.3 \\
\hline \multicolumn{16}{|c|}{ Praesepe } \\
\hline 41788 & 38 & 5.9 & 0.3 & 42327 & 37 & 5.7 & 0.3 & 42556 & 36 & 5.5 & 0.2 & 42952 & 36 & 5.3 & 0.3 \\
\hline 42133 & 37 & 5.7 & 0.3 & 42485 & 36 & 5.7 & 0.3 & 42578 & 36 & 5.3 & 0.2 & 42966 & 35 & 6.0 & 0.3 \\
\hline 42164 & 37 & 5.7 & 0.3 & 42516 & 36 & 5.4 & 0.2 & 42600 & 36 & 5.0 & 0.2 & 42974 & 35 & 5.8 & 0.3 \\
\hline 42201 & 37 & 5.6 & 0.3 & 42518 & 36 & 5.8 & 0.3 & 42673 & 36 & 5.7 & 0.3 & 43050 & 35 & 5.8 & 0.3 \\
\hline 42247 & 37 & 5.6 & 0.3 & 42523 & 36 & 5.7 & 0.3 & 42705 & 36 & 5.9 & 0.3 & 43086 & 36 & 5.6 & 0.3 \\
\hline 42319 & 37 & 5.7 & 0.3 & 42549 & 36 & 5.7 & 0.3 & 42766 & 36 & 5.7 & 0.3 & 43199 & 35 & 5.2 & 0.4 \\
\hline
\end{tabular}

Another complication is that, since some of the associations cover large areas of sky, there is an increased risk for contamination of the samples by field stars. Further, spectroscopic radial velocities often cannot be used to decide membership, both because they do not exist in significant numbers, and because their actual measurement is difficult for the often rapidly rotating $\mathrm{O}$ and B-type stars that make up much of these associations. For such reasons, the associations are here being treated separately.

The predictions in Paper I indicate that the accuracies of Hipparcos should enable astrophysically interesting results to be obtained for perhaps half a dozen of the nearer associations. Among these, Lower Centaurus Crux, Upper Centaurus Lupus and Upper Scorpius form part of the larger Scorpius OB2 complex, while the $\alpha$ Persei and "HIP 98321" associations are independent entities.

Except for "HIP 98321" (Sect. 5.6) the selection of members in the different associations is based on data from de Zeeuw et al. (1999). In their sample selection they combine one method using Hipparcos positions and proper motions (de Bruijne 1999a), and another using Hipparcos positions, proper motions and parallaxes (Hoogerwerf \& Aguilar 1999). Although this could cause some contamination by outliers, simulations showed that only $20 \%$ of the stars in the first method are expected to be field stars, and only $4 \%$ in the second. Although, in principle, our procedure for rejecting outliers does reduce this contamination, actually only few stars were rejected.

De Bruijne (1999b) used an implementation of our original method (Dravins et al. 1997) to obtain kinematically improved parallaxes for the three OB associations in the Scorpius OB2 complex (cf. Sect. 3.6). While the depth of the associations is not fully resolved by the Hipparcos parallaxes, the kinematically improved parallaxes reveal some internal structure. We refer to de Bruijne's work concerning the three-dimensional structure of the complex, although his distance estimates are slightly different from ours (mainly because his selection criterion, $g_{\text {lim }}=9$, differs from our $g_{\text {lim }}=15$ ). Based on the kinematically improved parallaxes, we presented the Hertzsprung-Russell diagrams of Upper Centaurus Lupus and Lower Centaurus Crux in Madsen et al. (2000). For additional discussion of the HR diagrams of the complex we again refer to de Bruijne (1999b).

The solutions for the associations as a whole were given in Table 1 (and its electronic version), while the results for the individual stars are given in the electronic version of Table 2.

\subsection{The Lower Centaurus Crux association}

The cleaned sample consists of 179 stars with an estimated internal dispersion of $1.1 \mathrm{~km} \mathrm{~s}^{-1}$. Combined with 
the rather small uncertainty of the cluster velocity, the resulting standard error for the astrometric radial velocities is 1.2 to $1.3 \mathrm{~km} \mathrm{~s}^{-1}$. In the corresponding HR diagram, the main sequence becomes somewhat better defined, most noticeably in the A-star regime (Madsen et al. 2000), although there still remains a significant spread.

\subsection{The Upper Centaurus Lupus association}

For Upper Centaurus Lupus, the rejection procedure produces a clean sample with 218 stars with an estimated internal dispersion of $1.2 \mathrm{~km} \mathrm{~s}^{-1}$. The resulting standard error of the astrometric radial velocities is $1.3 \mathrm{~km} \mathrm{~s}^{-1}$.

The HR diagram clearly shows an improvement across the whole spectral range of the main sequence (Madsen et al. 2000). Probably, the remaining spread is caused by non-detected binaries, some non-members, and premain sequence objects moving onto the main sequence. Differential reddening across the association and perhaps also in depth could also cause a spread of the main sequence, although Upper Centaurus Lupus is not believed to be as much affected as the other two associations in the Scorpius OB2 complex (de Zeeuw et al. 1999).

\subsection{The Upper Scorpius association}

The maximum-likelihood solution for the Upper Scorpius association became unstable after rejection of eight stars, at which point the criterion $g_{\max } \leq 15$ was still not met. We therefore choose to give results for the solution using all 120 stars in the original sample. The internal dispersion is in line with that of the previous two associations, but the larger uncertainty in the cluster velocity gives a higher standard error of about $1.9 \mathrm{~km} \mathrm{~s}^{-1}$ for the astrometric radial velocities.

It is difficult to judge whether the main sequence is actually better delineated by the kinematically improved parallaxes. Upper Scorpius appears to be close to the limit of our method, due to its larger distance, smaller angular size and a smaller number of member stars, compared with Lower Centaurus Crux and Upper Centaurus Lupus.

\subsection{The Scorpius OB2 complex}

Lower Centaurus Crux, Upper Centaurus Lupus and Upper Scorpius are all part of a larger OB complex, known as Scorpius OB2, with similar space velocity vectors (Blaauw 1964). Therefore, an attempt was also made to combine the three associations in a single solution assuming a common velocity vector. The resulting vector and internal dispersion are in Table 1, but we give no results for individual stars.

The HR diagram is visibly improved, indicating that Sco OB2 could meaningfully be regarded as one single structure. However, a combination of the HR diagrams from the three separate solutions is even slightly better defined, suggesting that Sco OB2 is, after all, better considered as three separate structures.

When treating Sco OB2 as one complex, the estimated internal velocity dispersion is only slightly larger than for the separate solutions, and the formal uncertainty of the space velocity vector is remarkably small. Nevertheless, when comparing the resulting astrometric radial velocities with those from the previous solutions we find noticeable differences. For Lower Centaurus Crux (LCC) we find $\left\langle v_{\mathrm{r} i}(\mathrm{LCC})-v_{\mathrm{r} i}(\mathrm{Sco} \mathrm{OB} 2)\right\rangle \simeq-2 \mathrm{~km} \mathrm{~s}^{-1}$, while for Upper Centaurus Lupus and Upper Scorpius the corresponding mean differences are $+4 \mathrm{~km} \mathrm{~s}^{-1}$ and $+10 \mathrm{~km} \mathrm{~s}^{-1}$, respectively. Such a progression of systematic differences could be expected if Sco OB2 is not really one uniform complex, or if there is some internal velocity field. At any rate, the comparison shows that one has to be careful when interpreting the results for young associations: although we get a stable solution with small residuals when considering the whole complex, the resulting velocities are not trustworthy.

Thus both the HR diagrams and the radial-velocity solutions indicate that the Sco OB2 complex has some internal kinematic structure that ultimately will need to be modelled, although it is only marginally discernible in the present data. In Sect. 5.7 we discuss the possible expansion of the associations.

\subsection{The $\alpha$ Persei association (Per OB3)}

This $\alpha$ Per association is sometimes denoted an open cluster. From our sample we obtain a mean astrometric radial velocity of $4.5 \pm 2.2 \mathrm{~km} \mathrm{~s}^{-1}$. A rather modest internal velocity dispersion $\sigma_{\mathrm{v}} \simeq 0.7 \mathrm{~km} \mathrm{~s}^{-1}$ was found using the procedure of Appendix A.4 in Paper II. The value is smaller than for the other OB associations, and indicates that it may be reasonable to look upon the structure as a young open cluster instead. The velocity dispersion, together with the uncertainty in the solution for the cluster velocity, combine to give a standard error of about $2.3 \mathrm{~km} \mathrm{~s}^{-1}$ in the astrometric radial velocities of the individual stars. The parallax improvement is not good enough to have a visible impact on the HR diagram.

Our radial-velocity result is close to the spectroscopic values of $\simeq+2 \mathrm{~km} \mathrm{~s}^{-1}$ (Prosser 1992), while somewhat larger than the $-0.9 \mathrm{~km} \mathrm{~s}^{-1}$ derived from the convergencepoint solution by Eggen (1998).

\subsection{The "HIP 98321" association}

This possible association was recently discovered in the Cepheus-Cygnus-Lyra-Vulpecula region by Platais et al. (1998), during a search for new star clusters from Hipparcos data. They named it after the central star HIP 98321, and found 59 probable members. Because of the Hipparcos limiting magnitude, only $\mathrm{O}, \mathrm{B}$, and A-type stars are utilizable. It was a bit surprising to find that this association gives a good kinematic solution despite 
its great distance of $307 \mathrm{pc}$; the reason is probably its large mean radius on the sky of $\sim 12$ degrees (Fig. 1).

The mean astrometric radial velocity is $-19.3 \pm$ $1.6 \mathrm{~km} \mathrm{~s}^{-1}$ for the sample of all 59 stars. Together with the estimated internal dispersion of $2.6 \mathrm{~km} \mathrm{~s}^{-1}$, the standard error of the individual astrometric radial velocities is around $3.2 \mathrm{~km} \mathrm{~s}^{-1}$. These values are consistent with the somewhat uncertain spectroscopic velocities for these early-type stars. Published values spread around $-15 \mathrm{~km} \mathrm{~s}^{-1}$, suggesting a possible expansion of the association compatible with its isochrone age (Table 4 in Paper I and next section).

During the cleaning process, the maximum $g$ value was always below $g_{\lim }=15$; thus no star was removed from the original sample. Some contamination by outliers may nevertheless be expected due to the lack of spectroscopic information in the selection of the stars. It would have been a nice confirmation of the existence of this new association if the improved parallaxes had given a better-defined main sequence, but unfortunately the improvement is not sufficient to have any visible effect in the HR diagram.

\subsection{Expanding associations?}

Figure 7 shows the astrometric versus spectroscopic radial velocities for stars in the Scorpius-Centaurus group of young associations, both for each individual subgroup, and for the complex treated as a whole. The spectroscopic values are those compiled in the Hipparcos Input Catalogue HIC (Turon et al. 1992). Because we are dealing with young and rapidly rotating early-type stars, the spectroscopic errors are quite large; some contamination is also expected due to outliers and binaries.

The astrometric radial velocities in $\mathrm{OB}$ associations are expected to show a significant bias due to expansion effects (Paper I). Assuming the inverse age of an association to be the upper limit on the relative expansion rate, the resulting maximum bias in the astrometric radial velocity can be computed from Eq. (10) in Paper I. This effect is directly proportional to the distance to the stars in the association and inversely proportional to its age. The expansion causes a positive shift in $v_{\mathrm{r}}($ spectr $)-\widehat{v}_{\mathrm{r}}$ (astrom): the cluster's increasing angular size is wrongly interpreted as approaching motion. This [upper limit of the] expansion bias is plotted in Fig. 7 together with the spectroscopic and astrometric velocities. We have not been able to observe any correlation between distance and expansion with the present data. The expected effect should be a few $\mathrm{km} \mathrm{s}^{-1}$, but it probably drowns in the noise from spectroscopic measurements that have errors of comparable magnitude, and from a possible anisotropic expansion.

The interpretation of Fig. 7 is not obvious. Stars in Lower Centaurus Crux show a wide spread in the spectroscopic values, while the mean is roughly consistent with an isotropic expansion at about half the rate naively expected from the age of the association. The same can be said for Upper Centaurus Lupus. For these associations the
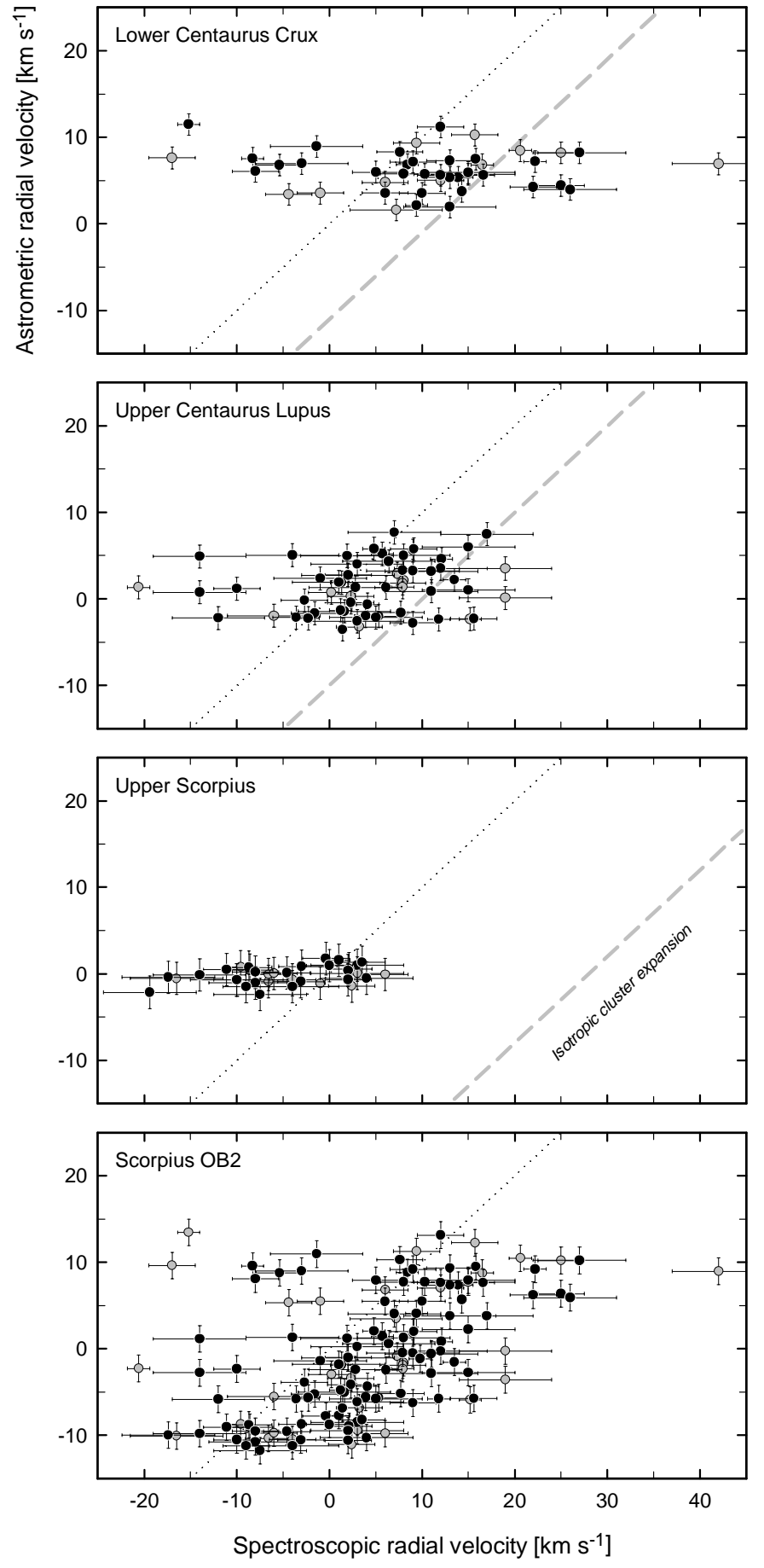

Fig. 7. Astrometric versus spectroscopic radial velocities for stars in the Scorpius-Centaurus group of young associations, expected to undergo kinematic expansion. The top three frames show separate solutions for each subgroup. Assuming a rate of isotropic expansion equal to the inverse age of the cluster, a bias in the astrometric radial velocity would result, marked by dashed gray lines (the cluster's increasing angular size would be interpreted as approaching motion; Paper I). The assumed ages are 11, 14 and 5 Myr, respectively (de Geus et al. 1989). The bottom frame shows the solution for all 510 stars in the groups, treated as one entity (only stars with known spectroscopic velocities are plotted). While these data do indicate some expansion of this complex of young associations, the expansion of its individual parts is significantly slower than the naively expected rate. 
indicated "kinematic age" (equal to the inverse of the current expansion rate) is thus around 20-30 Myr, or twice the isochrone ages according to de Geus et al. (1989). Upper Scorpius on the other hand, which is the youngest of the subgroups (5-6 Myr according to de Geus et al.), does not seem to expand at all: taken at face value, the data rather suggest that it contracts. The combined sample again indicates some expansion, roughly consistent with a kinematic age of $20 \mathrm{Myr}$. We note that already Blaauw (1964) derived such an expansion age of $20 \mathrm{Myr}$ for the Scorpius-Centaurus complex as a whole from the $\simeq 10 \mathrm{~km} \mathrm{~s}^{-1}$ discrepancy between the spectroscopic radial velocities and the proper motion data combined with photometric distances.

Of the three subgroups in Sco OB2, the result for Upper Scorpius thus stands out as rather puzzling. A detailed study of this association by Preibisch \& Zinnecker (1999) suggested that the star formation process was triggered by a giant supernova explosion in the neighbouring Upper Centaurus Lupus. What effect that may have had on the internal kinematics of Upper Scorpius is hard to say. There is a priori no reason to expect Upper Scorpius to be a bound system without expansion. The star formation in Upper Scorpius itself seems to have dispersed the rest of the parent molecular cloud. This result seems to imply the standard picture (see e.g. Mathieu 1986 for a review): the removal of gas leads to loss of binding mass of the system, it becomes unbound and consequently will expand.

From calculations inspired by our method, Makarov \& Fabricius (2001) estimated an expansion rate of $0.12 \mathrm{~km} \mathrm{~s}^{-1} \mathrm{pc}^{-1}$ for the TW Hya association of young stars, assuming a uniform expansion. The TW Hya association is dominated by late-type stars and may be an extension of Lower Centaurus Crux. The expansion corresponds to a bias of the centroid radial velocity of $\sim-9 \mathrm{~km} \mathrm{~s}^{-1}$ - comparable to the biases we find for Upper Centaurus Lupus and Lower Centaurus Crux - and to a dynamical age of 8.3 Myr, in agreement with previous age determinations for TW Hya's T Tauri members (Webb et al. 1999).

The results we find here are promising in the sense that it is possible to obtain information about the internal kinematics, formation history and age, but at the same time they confirm the complexity of the kinematics of the associations in the Sco OB2 complex. In the end more accurate spectroscopic observations are also required to answer these questions. These would in particular allow true expansion to be disentangled from the perspective effects of the radial motion.

\section{Conclusions}

The radial motions of stars have been studied through spectroscopy since the year 1868 (Hearnshaw 1986). Recently, the accuracies realized in astrometry have enabled such determinations to be made also through purely geometric methods. Once sufficient accuracies are reached, this will enable an absolute calibration of the stellar velocity scale for stationary and variable stars, irrespective of any complexities in their spectra. Indeed, for several early-type stars (with complex spectra smeared by their rapid rotation) the radial velocities already now determined through astrometry are more accurate than has been possible to reach spectroscopically in the past.

The differences between these astrometric radialvelocity values and wavelength measurements of different spectral features may become a new diagnostic tool in probing the dynamic structure of stellar atmospheres. Already the present work has made available quite accurate astrometric radial velocities for stars of many more spectral types than those for which hydrodynamic model atmospheres have been developed (from which, e.g., convective and gravitational wavelength shifts in their spectra could have been predicted). For such stars, the limitations in understanding the differences between astrometric and spectroscopic radial velocities may now lie primarily with spectroscopy and atmospheric modelling, rather than in astrometry.

In this series of three papers, we started by exploring different types of fundamental possibilities of astrometrically determining radial velocities, identifying which methods could be applicable on existing data already today. Among the latter, the moving-cluster method was found capable of yielding astrophysically interesting, sub-km s ${ }^{-1}$ accuracies, and its mathematical methods were developed in Paper II. In the present paper, data from Hipparcos were used in applying the method to obtain solutions for more than 1000 stars in nearby clusters and associations. Although most of these do not reach the high accuracies realized for the Hyades, they hint at the future potential.

Quantitatively, we have obtained radial velocities with standard errors of $\sim 0.6 \mathrm{~km} \mathrm{~s}^{-1}$ for individual stars in the Hyades. The accuracies reached begin to make visible the convective and gravitational shifts expected in the spectra of $\mathrm{F}$ and $\mathrm{G}$ stars. For A stars and earlier types, where the convective shifts cannot yet be reliably predicted from theory, the spectra appear to be blueshifted by a few $\mathrm{km} \mathrm{s}^{-1}$ compared with the astrometrically determined motions and expected gravitational redshifts. This illustrates that astrometric radial velocities with uncertainties even well in excess of $1 \mathrm{~km} \mathrm{~s}^{-1}$ could be astrophysically interesting.

Such accuracies may also be sufficient to provide information about the expansion of OB associations, as illustrated by the results for the Sco OB2 complex. Even with the modest precision of existing spectroscopic velocities, we see indications of expansion in the OB associations Upper Centaurus Lupus and Lower Centaurus Crux (causing a bias in the astrometric radial velocities of $5-10 \mathrm{~km} \mathrm{~s}^{-1}$ ), while Upper Scorpius surprisingly shows no such indication. The limitations in the present understanding of these associations come not from astrometry but mainly from spectroscopy and theory.

From the same solution that gave astrometric radial velocities, we get kinematically improved parallaxes. These can be used to study in greater detail the spatial 
structures and the Hertzsprung-Russell diagrams of both clusters and $\mathrm{OB}$ associations. In case of the Hyades, Upper Centaurus Lupus and Lower Centaurus Crux, the betterdefined main sequences can also be taken as proof of the validity of the kinematic solution, and hence of the astrometric radial velocities.

Hipparcos parallax measurements reached typical accuracies of about 1.5 mas, while our improved parallaxes reach 0.3 mas for the Hyades. Space astrometry missions in the near future are expected to improve this by more than an order of magnitude to about 0.05 mas (Horner et al. 1998), with another order-of-magnitude gain by the future GAIA to 0.004 mas (Perryman et al. 2001). As detailed in Paper I, such accuracies will enable also other methods than the moving-cluster one for determining radial velocities by purely geometric means. The future prospects for studying absolute radial velocities independent of spectroscopy look exciting indeed!

Acknowledgements. This project was supported by the Swedish National Space Board and the Swedish Natural Science Research Council. We want to thank Tim de Zeeuw for providing data on several nearby OB associations before publication, Floor van Leeuwen for providing data on the Pleiades and Praesepe clusters, and the referee, Anthony Brown, for valuable comments.

\section{References}

Andersen, J., \& Nordström, B. 1983, A\&A, 122, 23

Blaauw, A. 1964, ARA\&A, 2, 213

Castellani, V., Degl'Innocenti, S., \& Prada Moroni, P. G. 2001, MNRAS, 320, 66

de Bruijne, J. H. J. 1999a, MNRAS, 306, 381

de Bruijne, J. H. J. 1999b, MNRAS, 310, 585

de Bruijne, J. H. J., Hoogerwerf, R., \& de Zeeuw, P. T. 2000, ApJ, 544, L65

de Bruijne, J. H. J., Hoogerwerf, R., \& de Zeeuw, P. T. 2001, A\&A, 367, 111

de Geus, E. J., de Zeeuw, P. T., \& Lub, J. 1989, A\&A, 216, 44

de Zeeuw, P. T., Hoogerwerf, R., de Bruijne, J. H. J., Brown, A. G. A., \& Blaauw, A. 1999, AJ, 117, 354

Dravins, D. 2001, in Proc. 11th Cambridge Workshop on Cool Stars, Stellar Systems and the Sun, ed. R. J. García López, R. Rebolo, \& M. R. Zapatero Osorio, ASP Conf. Ser., 223, CD-778

Dravins, D., Lindegren, L., Madsen, S., \& Holmberg, J. 1997, in Hipparcos Venice' 97, ESA SP-402, ed. B. Battrick, \& M. A. C. Perryman (Paris: ESA), 733

Dravins, D., Gullberg, D., Lindegren, L., \& Madsen S. 1999a, in Precise Stellar Radial Velocities, ed. J. B. Hearnshaw, \& C. D. Scarfe, ASP Conf. Ser. 185, IAU Coll., 170, 41

Dravins, D., Lindegren, L., \& Madsen, S. 1999b, A\&A, 348, 1040 (Paper I)

Duflot, M., Figon, P., \& Meyssonnier, N. 1995, A\&AS, 114, 269

Eggen, O. J. 1984, AJ, 89, 1350
Eggen, O. J. 1998, AJ, 116, 1810

ESA 1997, The Hipparcos and Tycho Catalogues, ESA SP1200

Griffin, R. E. M., David, M., \& Verschueren, W. 2000, A\&A, 147,299

Griffin, R. F., Gunn, J. E., Zimmermann, B. A., \& Griffin, R. E. M. 1988, AJ, 96, 172

Gunn, J. E., Griffin, R. F., Griffin, R. E. M., \& Zimmermann, B. A. 1988 , AJ, 96,198

Hanson, R. B. 1975, AJ, 80, 379

Hearnshaw, J. B. 1986, The Analysis of Starlight: One hundred and fifty years of astronomical spectroscopy (Cambridge University Press, Cambridge), Chap. 6. (New edition 1990)

Høg, E., Fabricius, C., \& Makarov, V. V., et al. 2000, A\&A, $355, \mathrm{~L} 27$

Hoogerwerf, R., \& Aguilar, L. A. 1999, MNRAS, 306, 394

Horner, S. D., Germain, M. E., Greene, T. P., et al. 1998, BAAS, 193, 1206

Kovalevsky, J., Lindegren, L., Perryman, M. A. C., et al. 1997, A\&A, 323, 620

Jones, B. F., \& Walker, M. F. 1988, AJ, 95, 1755

Lebreton, Y. 2000, ARA\&A, 38, 35

Lebreton, Y., Fernandes, J., \& Lejeune, T. 2001, A\&A, 374, 540

Lindegren, L., Dravins, D., \& Madsen, S. 1999, in Precise Stellar Radial Velocities, ed. J. B. Hearnshaw, \& C. D. Scarfe, ASP Conf. Ser. 185, IAU Coll., 170, 73

Lindegren, L., Madsen, S., \& Dravins, D. 2000, A\&A, 356, 1119 (Paper II)

Madsen, S. 1999, in Harmonizing Cosmic Distance Scales in a Post-Hipparcos Era, ed. D. Egret, \& A. Heck, ASP Conf. Ser., 167, 78

Madsen, S., Lindegren, L., \& Dravins D. 2000, in Stellar Clusters and Associations: Convection, Rotation, ed. R. Pallavicini, G. Micela, \& S. Sciortino, ASP Conf. Ser., 198, 137

Makarov, V. V., \& Fabricius, C. 2001, A\&A, 368, 866

Makarov, V. V., Odenkirchen, M., \& Urban, S. 2000, A\&A, 358,923

Mathieu, R. D. 1986, Highlights of Astronomy, 7, 481

Montes, D. 2000, in Stellar Clusters and Associations: Convection, Rotation, ed. R. Pallavicini, G. Micela, \& S. Sciortino, ASP Conf. Ser., 198, 475

Narayanan, V. K., \& Gould, A. 1999, ApJ, 523, 328

Nelson, B., \& Young, A. 1970, PASP, 82, 699

Newberg, H. J., \& Yanny, B. 1998, ApJ, 499, L57

Nordström, B., Andersen, J., \& Andersen, M. I. 1997, A\&A, 322,460

Odenkirchen, M., Soubiran, C., \& Colin, J. 1998, New Astron., 3,583

Perryman, M. A. C., Brown, A. G. A., Lebreton, Y., et al. 1998, A\&A, 331, 81

Perryman, M. A. C., De Boer, K. S., Gilmore, G., et al. 2001, A\&A, 369, 339

Petrie, R. M. 1949, Publ. Dominion Astrophys. Obs., 8, 117

Petrie, R. M. 1963, Basic Astronomical Data, in Stars and Stellar Systems vol. II, ed. K. A. Strand (Univ. of Chicago Press, Chicago), 64

Pinsonneault, M. H., Stauffer, J., Soderblom, D. R., King, J. R., \& Hanson, R. B. 1998, ApJ, 504, 170

Pinsonneault, M. H., Terndrup, D. M., \& Yuan, Y. 2000, in Stellar Clusters and Associations: Convection, Rotation, ed. R. Pallavicini, G. Micela, \& S. Sciortino, ASP Conf. Ser., 198, 95 
Platais, I., Kozhurina-Platais, V., \& van Leeuwen, F. 1998, AJ, 116,2423

Portegies Zwart, S. F., McMillan, S. L. W., Hut, P., \& Makino, J. 2001, MNRAS 321, 199

Preibisch, T., \& Zinnecker, H. 1999, AJ, 117, 2381

Prosser, C. F. 1992, AJ, 103, 488

Rachford, B. L., Canterna, R. 2000, AJ, 119, 1296

Reid, I. N. 1996, AJ, 111, 2000

Rickman, H., ed. 2001, Transactions IAU vol. XXIV B, in press

Robichon, N., Arenou, F., Mermilliod, J.-C., \& Turon, C. 1999, A\&A, 345, 471

Schwan, H. 1991, A\&A, 243, 386,

Siess, L., Forestini, M., \& Dougados, C. 1997, A\&A, 324, 556

Soderblom, D. R., \& Mayor, M. 1993, AJ, 105, 226
Stello, D., \& Nissen, P. E. 2001, A\&A, 374, 105

Taylor, B. J. 1980, AJ, 85, 242

Tian, K. P., van Leeuwen, F., Zhao, J. L., \& Su, C. G. 1996, A\&AS, 118, 503

Turon, C., Crézé, M., Egret, D., et al. (eds.) 1992, The Hipparcos Input Catalogue, ESA SP-1136

van Leeuwen, F. 1999, A\&A, 341, L71

van Leeuwen, F. 2000, in Stellar Clusters and Associations: Convection, Rotation, ed. R. Pallavicini, G. Micela, \& S. Sciortino, ASP Conf. Ser., 198, 85

Webb, R. A., Zuckermann, B., Platais, I., et al. 1999, ApJ, 512, L63

Wielen, R. 1978, Mitt. Astron. Ges., 43, 261 